\title{
A FoxM1/IncRNA PTTG3P/YAP1 Positive Feedback Loop Controls Colorectal Cancer Progression
}

\section{Yang zheng}

Cancer Hospital of China Medical University, Liaoning Cancer Hospital \& Institute

\section{Guilin yu}

Cancer Hospital of China Medical University, Liaoning Cancer Hospital \& Institute

\section{Yiyang Liu}

Affiliated Hospital of Youjiang Medical University for Nationalities

\section{Longfei Xie}

University of California, Berkeley

Jinnian Ge

The central hospital of Shenyang medical college

\section{Yue wang}

Cancer Hospital of China Medical University, Liaoning Cancer Hospital \& Institute Guohua Zhao ( $\nabla$ glimmerbar@163.com )

Cancer Hospital of China Medical University, Liaoning Cancer Hospital \& Institute

\section{Research Article}

Keywords: Colorectal carcinoma, pseudogene, PTTG3P, FoxM1, YAP1

Posted Date: June 30th, 2021

DOI: https://doi.org/10.21203/rs.3.rs-587835/v1

License: (c) (1) This work is licensed under a Creative Commons Attribution 4.0 International License.

Read Full License 
A FoxM1/IncRNA PTTG3P/YAP1 positive feedback loop controls colorectal cancer progression

Yang zheng ${ }^{[1]}$, Guilin yu ${ }^{[2]}$, Yiyang Liu ${ }^{[3]}$, Longfei Xie ${ }^{[4]}$, Jinnian $\mathrm{Ge}^{[5]}$,Yue wang ${ }^{[2]}$, Guohua Zhao ${ }^{[2]}$

[1] Department of clinical laboratory, Cancer Hospital of China Medical University, Liaoning Cancer Hospital \& Institute, 110042 Liaoning, P. R. China

[2] Department of General Surgery, Cancer Hospital of China Medical University, Liaoning Cancer Hospital \& Institute, 110042 Liaoning, P. R. China

[3] Department of Surgery, Affiliated Hospital of Youjiang Medical University for Nationalities, 533000, Guangxi Zhuang autonomous region, P. R. China

[4] Department of Physics and Integrative Biology, University of California, Berkeley, CA 94720

[5] The central hospital of Shenyang medical college, 110031 Liaoning, P. R. China

Yue wang and Guohua Zhao are the co-corresponding authors.

Yang zheng and Guilin yu are the co-first authors.

Yue wang and Guohua Zhao are the co-corresponding authors.

Abstract Background Pseudogenes are vital regulators of cancer progression. PTTG3P biological function in colorectal cancer (CRC) needs further to be clarified. Methods qRT-PCR was adopted to measure the PTTG3P expression. Functional studies were examined by CCK-8, glucose uptake, lactate assay, ATP assay, ECAR assay and xenograft mice model. The mechanism of PTTG3P was carried by GSEA. Chromatin immunoprecipitation (ChIP) and luciferase assay were explored to certify the binding activity between PTTG3P promoter region and FoxM1. Results Ectopic expression of PTTG3P was involved in CRC and related to dismal prognosis. Experimental evidence discovered that PTTG3P enhanced cell proliferation and glycolysis through YAP1 and regulated FoxM1(Hippo pathway target gene). Further, LDHA knockdown or glycolysis inhibitor (2-DG,3-BG) recovered PTTG3P-induced proliferation. Mechanically, FoxM1 increase PTTG3P expression at transcription level and the PTTG3P promoter region of -900 to $-1200 \mathrm{nt}$ is necessary for binding with FoxM1, thus forming a positive feedback loop to facilitates the CRC progression. Additionally, FoxM1 depletion could rescue the PTTG3P function. Conclusions A FoxM1/lncRNA PTTG3P/YAP1 positive feedback loop plays a vital part in CRC progression, and targeting FoxM1, PTTG3P and YAP1 provides therapeutic targets for CRC treatment.

Key Words: Colorectal carcinoma, pseudogene, PTTG3P, FoxM1, YAP1

\section{INTRODUCTION}

Colorectal cancer (CRC) remains one of the major triggers of deaths from malignant tumors. Globally more than 1 million people suffer CRC every year[1]. As of 2012, CRC is the fourth cause of cancer death after lung, stomach, and liver cancer[2]. Despite improvements in diagnosis and combined treatment, patients with CRC have an even worse prognosis, especially in advanced patients. Therefore, it is quite urgent to clarify the mechanism, even potential approaches for the therapeutic intervention of CRC.

Accumulating evidence has shown that pseudogene, a type of long noncoding RNA, exhibits pivotal functions. It is estimated that the human genome has more than 18,000 pseudogenes. And pseudogene 
has emerged as key regulators of important biological processes involved in the development of human cancers. For instance, increased CYP4Z1 expression promotes tumor angiogenesis and growth in breast cancer partly via PI3K/Akt and ERK1/2 activation[3]. Nanog regulates primitive hematopoiesis by directly repressing critical erythroid lineage specifiers[4]. PTENP1 can exert a growth-suppressive role by regulating cellular levels of PTEN[5]. LncRNA PTTG3P (pituitary tumor-transforming 3, pseudogene, NR_002734), located at chromosome 8q13.1, was first reported in the study of human pituitary tumor transforming gene (hPTTG) family in 2000[6]. However, its biological function of the Warburg effect has yet to be illustrated in CRC.

Here, we presented that PTTG3P is overexpressed in CRC tissues and predicts a dismal prognosis. Enforced PTTG3P was linked with advanced clinical features. Functional experiments uncovered that PTTG3P promoted tumor growth and glycolysis via YAP1, then regulated Hippo pathway target gene FoxM1. Intriguingly, FoxM1 could increase PTTG3P expression and enriched in the promoter region of PTTG3P, forming a positive feedback loop.

\section{MATERIALS AND METHODS}

\section{Clinical samples}

120 patients with CRC were enrolled from the Affiliated Hospital of Youjiang Medical University for nationalities, the central hospital of Shenyang medical hospital, and Cancer Hospital of China Medical University between March 2010 and November 2015. The including criteria were as follow: patients were with a definite pathological diagnosis; No patients were received with chemotherapy or radiotherapy before surgery. The tumor and paired non-tumor tissues were also collected after lesion excision with $30 \mathrm{mins}$ and stored in liquid nitrogen, then transferred to a $-80^{\circ} \mathrm{C}$ refrigerator. And the characteristics of cases were thoroughly noted. All of the CRC patients have signed informed consent before utilizing the clinical resources for investigation aims. The study was approved by the Ethics Committee of Cancer Hospital of China Medical University.

\section{Cell lines culture}

Five human CRC cell lines (HT29, SW620, HCT-8, SW480, and HCT-116) and normal human intestinal epithelial cell lines (FHC, NCM460) were obtained from ATCC (Manassas, VA, USA). And cultured according to their instructions. All cells were cultured in an incubator according to their instructions at $37^{\circ} \mathrm{C}$ and in a humidified atmosphere with $5 \% \mathrm{CO} 2$.

\section{Total RNA isolation, qRT- PCR, and transfection}

The expression levels of RNA were calculated by the qRT-PCR system. Total RNA was extracted by TRIzol Reagent (Invitrogen), and $1 \mu \mathrm{g}$ of total RNA was reverse transcribed using the PrimeScript RT Reagent Kit (Perfect Real-Time; Takara). pcDNA3.1-PTTG3P, PTTG3P-containing lentiviral sequence vector (sh-PTTG3P) were purchased from GeneChem Corporation (Shanghai, China). CRC cells were transfected with plasmids in the presence of Lipofectamine 3000 (Invitrogen). After $48 \mathrm{~h}$ of transfection, cells were gathered for further use in the following experiments. The oligonucleotides were transfected into CRC cells using Lipofectamine 2000 (Invitrogen, USA) and the transfection efficiency was confirmed by qRT-PCR. The detail is in Table S1, S2.

\section{Cell proliferation assay}


Cell viability assay was carried out to analyze cell proliferation. Cell viability was estimated using CCK8 (CK04, DOJINDO, Beijing, China), based on the manufacturer's instruction. Cells were seeded in 96-well culture plates. After incubation for the indicated time, a CCK-8 reagent ( $10 \mu \mathrm{l})$ was added to each well. Cell viability was measured with a microplate reader for the absorbance at a wavelength of $450 \mathrm{~nm}$.

\section{Flow cytometry of apoptosis}

CRC cells in 6-well plates were rinsed in phosphate buffer saline (PBS), and then were trypsinized and resuspended in $100 \mu \mathrm{L}$ binding buffer added with $2.5 \mu \mathrm{L}$ of fluorescein isothiocyanate (FITC) conjugated Annexin V and $1 \mu \mathrm{L}$ of PI (Invitrogen). Fifteen minutes later, flow cytometry (BD Biosciences) was utilized for apoptotic cells.

\section{Glucose, lactate, adenosine triphosphate (ATP) levels, and extracellular acidification rate (ECAR)}

Glucose, lactate, adenosine triphosphate (ATP) levels, and extracellular acidification rate (ECAR). The levels of glucose and lactate were calculated with a Glucose Colorimetric Assay Kit (BioVision, CA) and a Lactate Assay Kit (BioVision, CA) in line with the instructions of the manufacturer. ATP level was tested using Cell Titer-Glo Luminescent Cell Viability Assay (Promega, Madison, MI). ECAR was detected using Seahorse XF 96 Extracellular Flux Analyzer (Agilent Technologies, Santa Clara, CA) according to the manufacturer's instructions.

\section{Databases used in the study}

PTTG3P expression in CRC cell lines was gathered by Cancer Cell Line Encyclopedia (CCLE) (www. broadinsti tute.org/ccle). Prediction of $\mathrm{CpG}$ islands in the PTTG3P promoter region was explored through online software (http://www.urogene.org/cgi-bin/methprimer) and DBCAT (http://dbcat.cgm.ntu. edu.tw/). DNA methylation analysis of CRC samples was carried out by the SMART App (http://www. bioinfo-zs.com/smartapp) which is based on the UCSC Xena public data of TCGA (https://xenabrowser.net).

\section{Dual-luciferase assay}

The complementary DNA fragment containing the wildtype or mutant PTTG3P fragment and 3'-UTR of FoxM1 was subcloned downstream of luciferase gene within the pGL3-Basic luciferase reporter vector (Promega, USA). To determine FoxM1 as an upstream regulator of PTTG3P, CRC cells were transfected with reporters, siRNAs, or specific gene expression plasmids. Relative luciferase activities of 3'- UTR of FoxM1 and PTTG3P were normalized to Renilla luciferase activity at 24h after transfection.

\section{Chromatin Immunoprecipitation assay (ChIP)}

The experiments were performed according to previous reports. CRC cells $(2 \times 106)$ were prepared for ChIP assay using a ChIP assay kit (Millipore) according to the manufacturer's protocol. The resulting precipitated DNA specimens were analyzed using PCR to amplify fractions of the PTTG3P promoter. The PCR products were resolved electrophoretically on a $2 \%$ agarose gel and visualized using ethidium bromide staining. 


\section{Drug treatment}

FDI-6 (Axon2384; Axon Medchem) was used with a concentration of $10 \mathrm{mM}$. Cells were treated with the respective drugs at 12, 24, 48 and $72 \mathrm{~h}$; the drug was then washed out for the remainder of the culture time. Experiments were performed in triplicates.

\section{Animal study}

HCT-116 cells were transfected with sh-PTTG3P. $1 \times 107$ indicated cells were subcutaneously injected into 4-week-old male nude mice. Tumor volume measured every 5 days. 2-DG using in the study was injected into the abdominal cavity ( $1000 \mathrm{mg} / \mathrm{kg}$, injected into the abdominal cavity). Oxaliplatin using in the study was injected into the abdominal cavity ( $5 \mathrm{mg} / \mathrm{kg}$ twice per week). After 35 days, the twenty mice were sacrificed, and the tumor weight was measured. The animal study was carried out following the Guide for the Care and Use of Laboratory Animals of the NIH. This study had been approved by the Committee on the Ethics of Animal Experiments of Youjiang Medical University for nationalities and China medical university.

\section{Statistical analysis}

All the data were shown as the mean \pm standard deviation, at least three independent experiments. The difference between two independent groups was analyzed by a two-tailed Student's t-test, while multigroup comparison was made by ANOVA. Expression correlation between genes was analyzed by Pearson correlation analysis. Survival analysis was conducted using the Kaplan-Meier method and analyzed by the log-rank test. SPSS 22.0 (SPSS Inc., Chicago, IL, USA) was used to conduct statistical analyses, and differences were ensured when P-value was $<0.05$.

\section{RESULTS}

\section{PTTG3P is highly expressed in CRC}

To evaluate potential lncRNAs involved in mediating CRC progression, we examined the lncRNA expression profile (GSE 84983) (Fig. S1a). Comparison between CRC tumor tissues and adjacent normal tissues, we focused on the upregulated lncRNAs (fold change $>5, \mathrm{P}<0.01$ ), for these lncRNAs might be oncogenes and therapeutic targets. LncRNA PTTG3P was one of the most upregulated and chosen for consideration (Fig. S1b). Then, we found that PTTG3P had rarely the ability to code proteins, using the open-reading frames (ORFs) Finder and conserved domain database. Moreover, five other different online metrics got the same conclusion (Table S3). Additionally, we identified no valid Kozak consensus sequence in PTTG3P[7], indicating that PTTG3P was a long noncoding RNA with no protein-coding potential. Then we explored the subcellular location of PTTG3P by using lncRNA subcellular localization predictor software (lncLocator, http://www.csbio.sjtu.edu.cn/bioinf/lncLocator/) (Fig.S1c), suggesting PTTG3P was mainly localized to the cytoplasm, and subcellular fractionation confirmed the prediction (Fig. S1d).

To verify the elevation of PTTG3P in CRC, we investigated the detailed annotative process of preclinical human cancer models via the Cancer Cell Line Encyclopedia (CCLE) (www.broadinstitute.org/ccle), indicating that PTTG3P was remarkably overexpressed in cell lines of CRC (Fig. 1a, 1b). Then, the cell lines of HT-29, SW620, HCT-8, SW480, HCT116, NCM460, and FHC were conducted for PTTG3P expression. As showed in Fig. 1c, the PTTG3P expression was exceedingly increased in HT-29, SW620, HCT-8, SW480, HCT116 cells, compared with NCM460 and 
FHC cells. Further, we explored PTTG3P expression in a cohort of 120 paired and non-tumor tissues of CRC, the clinicopathologic characteristics are demonstrated in Table 1. Significantly, the PTTG3P level was overexpressed in CRC tissues compared to their counterparts (Fig. 1d, 1e), which was in accordance with the results of the TCGA database (Fig. 1f, 1g). Besides, high PTTG3P expression was observed in other malignant tumors (Fig. 1h). Also, our specimens confirmed PTTG3P overexpression in stomach adenocarcinoma (STAD), and esophageal squamous cell carcinoma (ESCA) (Fig. 1i,1j). Intriguingly, there was no actionable EGFR, VEGFR or RAS mutations, indicating that higher expressed PTTG3P may be driven by oncogenic event (Fig. S1e-1g). Altogether, these data revealed that PTTG3P was elevated in CRC and might be an oncogene.

\section{High PTTG3P level correlates with poor prognosis}

To identify the connection between the level of PTTG3P and clinicopathologic features, we divided the cases into PTTG3P low-expression and high-expression groups based on the median expression. Upregulated PTTG3P was positive linked with Tumor size $(\mathrm{P}=0.02)$ and Differentiation $(\mathrm{P}=0.01)$, but not with age $(\mathrm{P}=0.86)$, gender $(\mathrm{P}=0.74)$, tumor invasion depth $(\mathrm{P}=0.28)$, lymph node metastasis $(\mathrm{P}=0.09)$ or vessel invasion ( $\mathrm{P}=0.06)$ (Table 1). Moreover, the PTTG3P expression in stage III-IV was higher than that in stage I-II tissues, and PTTG3P was expressed much more highly in advanced CRC samples than early CRC tissue (Fig. 2a). Additionally, Kaplan-Meier survival curves illustrated that patients with highly expressed PTTG3P had poorer survival time (Fig. 2b). Further, we determined the prognostic ability of PTTG3P in CRC. As shown in Table 2, univariate analyses suggested highly expressed PTTG3P was associated with a dramatic risk of death $(\mathrm{P}<0.01)$. Multivariate analysis demonstrated that PTTG3P expression was an independent prognostic factor $(\mathrm{P}<0.01)$. A model that incorporated the independent predictor was proposed as the nomogram (Fig.S2). Subsequently, the ROC curve was carried out to evaluate the diagnostic capacity of PTTG3P in CRC tissues compared with normal counterparts, the area under the ROC curve (AUC) was 0.776 (95\% CI 0.733-0.819) (Fig. 2c). Thus, these data suggested that high expression of PTTG3P predicted a worse prognosis and may serve as a clinical biomarker for CRC patients.

\section{PTTG3P is caused by metabolic stress and promotes glycolysis and proliferation in CRC}

To investigate the biological function of PTTG3P, we transfected the PTTG3P overexpressed plasmids and shRNA targeting PTTG3P into HT-29 and HCT116 cells, respectively (Fig. 2d). By determining PTTG3P expression via gene set enrichment analysis (GSEA) the Cancer Genome Atlas (TCGA) profiles, we found that PTTG3P level was positively correlated with the glycolysis by affecting genes in glycolysis regulation (Fig. 2e). PTTG3P knockdown restrained the mRNA level of GLUT-1, ALDOA, PKM2, and LDHA, and the effect of silenced PTTG3P on glycolytic gene transcription could be rescued by PTTG3P re-expression (Fig. 2f).

And glucose deprivation is a well-known feature of solid tumors. Subsequently, we wonder whether PTTG3P participated in cell survival under metabolic stress, then we carried out several experiments with different glucose concentrations and glycolysis inhibitor 2-deoxyglucose (2-DG) to make a condition of glucose deprivation. Obviously, PTTG3P expression was increased by glucose deprivation or 2-DG treatment in either dose-dependent or time-dependent manner (Fig. 2g, 2h). Thus, we elucidated that PTTG3P could play a crucial role in the progression of metabolic stress. 
Next, we performed the glucose uptake analysis, ATP analysis, lactate production analysis, and discovered that PTTG3P knockdown repressed these phenomena. In contrast, PTTG3P overexpression boosted glucose uptake (Fig. 3a), lactate production (Fig. 3b), and ATP accumulation (Fig. 3c). Additionally, we calculated the level of ECAR, PTTG3P depletion notably hindered glycolytic capacity and vice verse (Fig. 3d). Also, we found that PTTG3P silencing suppressed the proliferation, facilitated apoptosis of HCT116 cells, whereas upregulated PTTG3P increased the proliferation, inhibited apoptosis of HT-29 cells according to the CCK-8 assay and flow cytometry analysis (Fig. 3e,3f). In vivo, highly expressed PTTG3P efficiently increased the tumor growth (Fig. 3g,3h). We then explored whether glycolysis played a vital role in cell proliferation and tumor growth. Notably, the glycolic inhibitors 2-DG and 3-BP or depletion of LDHA, which catalyzed the final step of glycolysis, could partly abrogate cancer cell proliferation and tumor growth (Fig. 3i,3j,3k). Clinically, Oxaliplatin is used for the treatment of colorectal cancer. Previously, it is reported that suppression of glycolysis is an effective strategy to block cell proliferation and conquer drug resistance. As shown in Fig. 31,3m, PTTG3P depletion and Oxaliplatin played a synergistic role in emancipating tumor growth. As a taken, PTTG3P knockdown plus Oxaliplatin is a promising therapy for CRC.

\section{PTTG3P regulates Hippo signaling pathway in CRC}

In order to elucidate which pathway involved in PTTG3P-meddated CRC progression, GSEA in the published TCGA CRC database was explored. And we suggested that PTTG3P expression was associated with the YAP1-activated gene signatures, indicating that Hippo signaling pathway may participated in the biological function of PTTG3P (Fig. 4a). To verify the speculation, the hub genes in Hippo pathway, including LATS1/2, MST1/2 and YAP1, and Hippo pathway target genes, such as CDX2, FOXM1, CTGF and CYR61, were tested in sh-PTTG3P HCT-116 cells. Subsequently, PTTG3P knockdown impaired the mRNA level of YAP1, FOXM1 and CTGF (Fig. 4b). It is commonly acknowledged that YAP1, a crucial factor in Hippo pathway, involves in cell proliferation. In our study, the level of PTTG3P and YAP1 displayed positive linkage in CRC tissues (Fig. 4c), and YAP1was highly expressed in CRC (Fig. S1h, S1i), associated with advanced characteristics of CRC (Table S4). Further YAP1 had a high diagnostic value with AUC 0.793(95\% CI 0.729-0.858) from TCGA database (Fig. S1j)..

Besides, we performed rescue assays in HT-29 cells. PTTG3P overexpression plus YAP1 knockdown could reverse PTTG3P induced phenotype (Fig. 4d-4g). Intriguingly, the treatment of Hippo pathway inhibitor, XMU-MP-1 (inhibiting MST1/2), could not recover the promoting effect of PTTG3P on proliferation, apoptosis and tumor growth (Fig. 4h-4k). In brief, all the data uncovered that PTTG3P hedges the key factor MST1/2, while modulates YAP1 in Hippo pathway to exhibit pivotal functions in CRC progression.

\section{Methylation and deacetylation are not involved in PTTG3P upregulation in CRC}

We first determined whether DNA methylation can regulate PTTG3P expression. No CpG islands were found in the PTTG3P promoter, as shown by analyzing PTTG3P promoter sequences via the online software MethPrimer (http://www.urogene.org/cgi-bin/methprimer/methprimer.cgi) and DBCAT (http://dbcat.cgm. ntu.edu.tw/) (Fig. 5a, b). To validate the role of DNA methylation on the regulation of PTTG3P expression, HT29 and HCT-116 cells were transfected with small interfering RNA (siRNA) for DNA (cytosine-5)-methyltransferase 1,3A,3B (DNMT1,3A,3B), crucial enzymes catalyzing the 
transfer of methyl groups to certain $\mathrm{CpG}$ structures of DNA. Our results demonstrated that these DNA methylation enzymes could barely influence PTTG3P expression(Fig. 5c). Additionally, we treated CRC cells with $5 \mu \mathrm{M}$ 5-azacytidine (5-AZA), an inhibitor of DNA methylation, our results revealed that 5-AZA treatment did not affect PTTG3P levels in CRC cells (Fig. 5d). Accumulating evidence has shown that ectopic expression of IncRNAs could be regulated by transcriptional factors, and histone acetylation plays a critical role in this procession. Then, we carried out experiments with SAHA and $\mathrm{NaB}$, the broad-spectrum HDAC inhibitors, and we discovered that these HDAC inhibitors failed to alter PTTG3P level in HT29 cells (Fig. 5e). In addition, overexpressed HDAC6 and HDAC8 did not affect increasing PTTG3P expression (Fig. 5f). Next, histone methylation may also influence gene transcription. We knocked down EZH2 and LSD1 in HT29 and HCT-116 cells and measure the PTTG3P expression. As a result, silencing EZH2 or LSD1 did not markedly influence PTTG3P expression, indicating that histone methylation is not involved in PTTG3P upregulation in CRC cells (Fig. 5g). In summary, our findings preliminarily demonstrated that methylation and deacetylation are not participating in the upregulation of PTTG3P in CRC.

\section{FoxM1 regulates PTTG3P expression and rescues the function of PTTG3P}

TFs are fundamental players in regulating gene expression. Consequently, we hypothesized that the elevated PTTG3P might be induced by the transcription factor. According to the JASPAR and UCSC website analysis, we suggested that FoxM1 might play a critical role by binding to the PTTG3P promoter. Firstly, FoxM1 was associated with advanced characteristics of CRC (Table S5) and was highly expressed in CRC and other malignant tumors (Figure S3b-3e). Further FoxM1 had a high diagnostic value with AUC 0.926 (95\% CI 0.897-0.955) from the TCGA database (Figure S3a). Secondly, we knocked down FoxM1 in 2 cell lines with 2 targets and selected si-FoxM1(1) for the next study (Figure 6a). PTTG3P levels were reduced concomitant with the downregulation of FoxM1 using siRNA, and subsequent re-expression of FoxM1 could recover the PTTG3P expression (Figure 6b). Besides, treatment with inhibitors of FoxM1 ( FDI-6 ), remarkably counteracted FoxM1-induced PTTG3P expression (Figure S4a). To figure out whether these inhibitors could impede FoxM1 binding in HCT-116 cells, which highly express FoxM1, we selected RCM-1 and FDI-6 for follow up experiments. ChIP analysis indicated that the enrichment of FoxM1 binding was decreased in PTTG3P promoter region with FDI-6 (20 $\mu \mathrm{M}, 6 \mathrm{~h})$ not RCM-1 (Figure S4b). As recently reported that activation of CCNB1 and CDC25 genes has been attributed to the oncogenic potential of FoxM1 $[8,9]$, thus we explored ChIP with FoxM1 antibody and FDI-6 $(20 \mu \mathrm{M}, 6 \mathrm{~h})$ followed by qRT-PCR amplification of the bound DNA. This revealed the diminished level of FoxM1 occupancy at CCNB1 and CDC25B promoter region with FDI-6 (Figure S4c). Thirdly, to confirm that PTTG3P is a transcriptional target of FoxM1, we cloned serialized truncations of PTTG3P promoter into the pGL3-basic vector and measured the luciferase activity after transfecting them into HEK-293T cells. Our results demonstrated that the luciferase activities were presented in -1200 to $0 \mathrm{nt}$, not -900 to $0 \mathrm{nt}$, indicating PTTG3P promoter region $(-1200 \sim-900)$ is necessary for FoxM1 interaction (Figure 6c).

Fourthly, luciferase reporter assay confirmed that the reduced FoxM1 level could weaken the PTTG3P promoter activity (Figure 6d), ChIP analysis indicated that the enrichment of FoxM1 binding was obvious in the PTTG3P promoter region (-900 to -1200 nt), suggesting that FoxM1 modified PTTG3P level at the transcriptional level (Figure 6e). Finally, we explored that whether the promoter region ( -900 to $-1200 \mathrm{nt}$ ) was crucial for PTTG3P biological function. We then overexpressed truncation (-900 
to $-1200 \mathrm{nt}$ ) in HT29 cells. However, PTTG3P truncation (-900 to $-1200 \mathrm{nt}$ ) could barely promote glucose uptake (Figure 6f), cell proliferation (Figure 6g) and inhibited apoptosis (Figure 6h). Also, PTTG3P truncation (-900 to -1200 nt) partly recovered the sh-PTTG3P regulating gene expression with no statistical significance (Figure 6i, 6j). Besides, FoxM1 depletion could recover the PTTG3P function (Fig. 6k-6o). Collectively, A FoxM1/lncRNA PTTG3P/YAP1 positive feedback loop is vital for CRC progression (Figure 6p).

\section{PTTG3P plays crucial functions in CRC immunology}

Cancer cells have high glucose uptake and glycolysis, resulting in a low level of glucose in the tumor, thus inhibiting the production of IFN-r by CD8+T cells in the tumor[10]. The tumor immune environment broadly participates in different malignant tumors, including CRC. Recently, the treatment of CRC with immune checkpoint inhibitors (ICI) has provided a potential clinical treatment. Interestingly, our findings proposed that low PTTG3P expression relates with CD8+ T, NK and TFH cells infiltration in the microenvironment of CRC, not with Treg or macrophages infiltration, based on the TCGA database Figure S4d-i). And the results of ELISA showed that the level of inflammatory cytokines TNF- $\alpha$, IL-1 $\beta$ and IL-6 were decreased with PTTG3P depletion (Figure S4j).

\section{DISCUSSION}

Pseudogene may be transcribed into RNA at low levels, due to promoter elements inherited from the ancestral gene or arising by new mutations. Although most transcripts have rarely functional significance than chance transcripts from other parts of the genome, some have given rise to regulatory RNAs and new proteins. For instance, long noncoding RNA HK2P1, a pseudogene of HK2, promoted lactate production and glucose uptake in endometrial stromal cells[11]. Pseudogene PTENP1 repressed the oncogenic PI3K/AKT pathway and inhibited the HCC progression[12]. To date, the role of pseudogene PTTG3P in glycolysis of CRC has not been reported yet. Our findings documented that PTTG3P facilitated CRC progression via the FoxM1/PTTG3P/YAP1 axis.

Our study verified that PTTG3P is highly expressed and has a potential diagnostic value, with an AUC of 0.776 (95\% CI 0.733-0.819) in CRC. Clinically, high PTTG3P expression considerably associates with tumor size and TNM stage as well as shorter survival time. These results confirmed that PTTG3P serves as a valuable prognostic biomarker and aids innovatively efficient therapies for CRC patients. Additionally, our findings stand in line with other research, Liu, et al.[13] reported that PTTG3P was remarkably upregulated in CRC tumor samples than that in normal samples. Zhou, et al.[14] revealed that PTTG3P is a valuable resource for identification in HCC progression and is useful for biomarker development. Weng, et al.[15] certified that PTTG3P facilitates cell proliferation, migration and invasion and might serve as a new promising strategy for gastric cancer. Recently, PTTG3P expression has a relationship with breast cancer[16] and pancreatic cancer[17]. Thus, the oncogenic role of PTTG3P in malignant tumors is strongly suggested.

Malignant tumors could undergo glycolysis at a higher speed than that of non-tumor tissue controls[18-20]. This phenomenon is known as the Warburg effect[21]. The Warburg hypothesis demonstrates that malignant tumor is fundamentally caused by mitochondrial metabolism disorder. Doherty JR, et al.[20] found that tumor lactate levels correlate with increased metastasis, tumor recurrence, and poor outcome. And targeting lactate metabolism is a prospective method for cancer 
therapeutics. Furthermore, cancer cells with high level of glycolysis and acid resistance have an energetic growth advantage, which facilitates unrestrained proliferation and invasion. In our study, we explored gain and loss-of-function approaches in HT29 and HCT-116 cells and found PTTG3P ablation resulted in the inhibition of CRC cell glycolysis by regulating numerous genes linked with metabolic pathways, whereas the opposite outcome was observed after enforced expression of PTTG3P. Nowadays, the ketogenic diet was used to constrain glycolysis to starve cancer cells, adjusting mitochondrial metabolism[22]. Here, we also proposed that the biological mechanism of PTTG3P on boosting cell proliferation might resist apoptosis.

Hippo signaling pathway has become increasingly important in human cancer[23], the key regulator YAP1 has been certified to be upregulated in breast cancer, colorectal cancer, and liver cancer[24], and YAP1 could promote cell growth[25-27] and inhibit apoptosis[28]. Clinically, YAP1 could be a target for the development of cancer drugs[29]. Yi, et al. [30] suggested that inhibiting TEAD-YAP1 interactions or block the binding function of WW domains is a pharmacologically viable strategy against the YAP1 oncoprotein. In our presented study, we discovered that PTTG3P activates Hippo signaling pathway by promoting YPA1, FoxM1 and CTGF, not MST1/2, and rescue assay consolidates this by using the Hippo pathway inhibitor, XMU-MP-1 (inhibiting MST1/2).

Then, we investigated the mechanism responsible for PTTG3P upregulation in CRC and found transcription factor FoxM1 increase PTTG3P expression at the transcription level. The promoter region ( -900 to -1200$)$ of PTTG3P is essential for binding with FoxM1 and the PTTG3P regulation of glycolysis and proliferation. Additionally, we carried out a series of experiments with FoxM1 inhibitors (RCM-1 and FDI-6 ) to test and verify the regulation and function of FoxM1. Our data certified that FDI-6 could decrease the binding FoxM1 in PTTG3P promoter and the known downstream genes of CCNB1 and CDC25B expression at the transcription level. A growing amount of lncRNAs play a vital part in FoxM1- induced signaling way. Zhi, et al. [31] identified the potential reciprocal link between LINC-ROR and FoxM1 and demonstrated that this correlation was involved in intrinsic resistance to sorafenib. And Li, et al.[32] indicated that the enhancement of FoxM1 by AKT in osteosarcoma cells activates LINC-TUG1 transcription by direct binding to the TUG1 promoter. Then, Chen, et al. Proposed that FoxM1 directly binds to the promoters of linc-Snhg8 and linc-Gm26917 to increase the expression.

Besides, our findings from the TCGA database proposed that low PTTG3P expression relates with CD8+ T, NK and TFH cells infiltration in the microenvironment of CRC, not with Treg or macrophages infiltration. And we found that a decreased TNF- $\alpha$, IL- $1 \beta$ and IL-6 level was accompanied by ablation of PTTG3P.

\section{CONCLUSION}

In conclusion, our data proposed that PTTG3P is upregulated in CRC cell lines and tissues, and is related to poor prognosis in CRC patients. PTTG3P promotes cell proliferation, glycolysis, and boosts tumorigenesis and increases the TNF- $\alpha$, IL- $1 \beta$ and IL- 6 level, because of suppressing CD8+ T, NK and TFH cell infiltration. Moreover, our present findings discovered that the transcription factor FoxM1, not methylation nor deacetylation, increases PTTG3P level in CRC. PTTG3P promoter region (-900 to -1200 ) is crucial for mediating oncogenic bioeffects and for binding with FoxM1. Hence, PTTG3P and 
FoxM1 might be together used as significant targets for CRC prevention and therapy, shedding some light on the poorly understanding of pseudogene in cancer biology.

\section{Reference}

[1] Cunningham D, Atkin W, Lenz HJ, et al. Colorectal cancer. Lancet.2010, 375 (9719): 1030-1047.

[2] Adelstein BA, Macaskill P, Chan SF, et al. Most bowel cancer symptoms do not indicate colorectal cancer and polyps: a systematic review. BMC Gastroenterology. 2011,11: 65.

[3]Michael A Rieger, Reinhard Ebner, David R Bell, et al. Identification of a novel mammary-restricted cytochrome P450, CYP4Z1, with overexpression in breast carcinoma. Cancer Res.2004, 64, 2357-2364.

[4] Wei Yu, Hongyan Chai, Ying Li, et al. Increased expression of CYP4Z1 promotes tumor angiogenesis and growth in human breast cancer. Toxicol Appl Pharmacol. 2012,264(1): 73-83.

[5] Laura Poliseno, Leonardo Salmena, Jiangwen Zhang, et al. A coding-independent function of gene and pseudogene mRNAs regulates tumour biology. Nature. 2010,24; 465(7301): 1033-1038.

[6] Chen L, Puri R, Lefkowitz EJ, et al. Identification of the human pituitary tumor transforming gene (hPTTG) family: molecular structure, expression, and chromosomal localization. Gene. 2000;248:41-50.

[7] Kozak M. Point mutations define a sequence flanking the AUG initiator codon that modulates translation by eukaryotic ribosomes. Cell. 1986;44: 283-292.

[8] Gillian M Keating. Sorafenib: A Review in Hepatocellular Carcinoma.Target Oncol. 2017, 12(2):243-253.

[9] Augusto Villanueva, Josep M Llovet. Targeted therapies for hepatocellular carcinoma.

Gastroenterology. 2011,140(5):1410-1426.

[10] Chang CH, Qiu J, O'Sullivan D, et al. Metabolic Competition in the Tumor Microenvironment Is a Driver of Cancer Progression. Cell 2015; 162(6): 1229-1241.

[11] Hong Lv, Jing Tong, Jieqiong Yang, et al. Dysregulated Pseudogene HK2P1 May Contribute to Preeclampsia as a Competing Endogenous RNA for Hexokinase 2 by Impairing Decidualization. Hypertension. 2018,71(4):648-658.

[12] Chiu-Ling Chen, Yen-Wen Tseng, Jaw-Ching Wu, et al. Suppression of hepatocellular carcinoma by baculovirus-mediated expression of long non-coding RNA PTENP1 and MicroRNA regulation. Biomaterials. 2015,44:71-81.

[13] Liu N, Dou L, Zhang X. LncRNA PTTG3P Sponge Absorbs microRNA-155-5P to Promote Metastasis of Colorectal Cancer. 2020,(9)13:5283-5291.

[14] Zhou Q, Zhang W, Wang Z,et al. Long non-coding RNA PTTG3P functions as an oncogene by sponging miR-383 and up-regulating CCND1 and PARP2 in hepatocellular carcinoma. BMC Cancer. 2019, 24;19(1):731.

[15] Weng W, Ni S, Wang Y, et al. PTTG3P promotes gastric tumour cell proliferation and invasion and is an indicator of poor prognosis. J Cell Mol Med. 2017,21(12):3360-3371.

[16] Lou W, Ding B, Fan W. High Expression of Pseudogene PTTG3P Indicates a Poor Prognosis in Human Breast Cancer. Mol Ther Oncolytics. 2019,27;14:15-26.

[17] Liu W, Tang J, Zhang H, et al. A novel lncRNA PTTG3P/miR-132/212-3p/FoxM1 feedback loop facilitates tumorigenesis and metastasis of pancreatic cancer. Cell Death Discov. 2020,30;6(1):136.

[18] Robert A Gatenby, Robert J Gillies. Why do cancers have high aerobic glycolysis?. Nat Rev Cancer, 2004, 4(11): 891-899. 
[19] Juanjuan Ou, Hongming Miao, Yinyan Ma, et al. Loss of abhd5 promotes colorectal tumor development and progression by inducing aerobic glycolysis and epithelial-mesenchymal transition. Cell Rep, 2014, 9(5): 1798-1811.

[20] Joanne R Doherty, John L. Cleveland Targeting lactate metabolism for cancer therapeutics. J Clin Invest, 2013, 123(9): 3685-3692.

[21] WARBURG O. On the origin of cancer cells. Science, 1956, 123(3191): 309-314.

[22] Laurent Schwartz, Thomas Seyfried, Khalid O Alfarouk, et al. Out of Warburg effect: An effective cancer treatment targeting the tumor specific metabolism and dysregulated $\mathrm{pH}$. Semin Cancer Biol, 2017, 43:134-138.

[23] Leslie J Saucedo, Bruce A Edgar. Filling out the Hippo pathway. Nat Rev Mol Cell Biol. 2007, 8(8):613-621.

[24] Zender L, Spector MS, Xue W, et al. Identification and validation of oncogenes in liver cancer using an integrative oncogenomic approach.Cell. 2006,30; 125(7): 1253-1267.

[25] Shimomura T, Miyamura N, Hata S, et al. The PDZ-binding motif of Yes-associated protein is required for its co-activation of TEAD-mediated CTGF transcription and oncogenic cell transforming activity. Biochemical and Biophysical Research Communications. 2014,443 (3): 917-923.

[26] Harvey KF, Zhang X, Thomas DM. The Hippo pathway and human cancer. Nature Reviews. Cancer. 2013,13 (4): 246-257.

[27] Johnson R, Halder G. The two faces of Hippo: targeting the Hippo pathway for regenerative medicine and cancer treatment. Nature Reviews. Drug Discovery. 2014,13 (1): 63-79.

[28] Zhao B, Kim J, Ye X, et al. Both TEAD-binding and WW domains are required for the growth stimulation and oncogenic transformation activity of yes-associated protein. Cancer Research.

2009, 69 (3): 1089-1098.

[29] Sudol M, Shields DC, Farooq A. Structures of YAP protein domains reveal promising targets for development of new cancer drugs. Seminars in Cell \& Developmental Biology. 2012, 23 (7): 827-833.

[30] Liu-Chittenden Y, Huang B, Shim JS, et al. Genetic and pharmacological disruption of the TEAD-YAP complex suppresses the oncogenic activity of YAP. Genes \& Development. 2012, 26 (12): 1300-1305.

[31] Yingru Zhi, Mubalake Abudoureyimu, Hao Zhou, et al. FOXM1-Mediated LINC-ROR Regulates the Proliferation and Sensitivity to Sorafenib in Hepatocellular Carcinoma. Mol Ther Nucleic Acids. 2019, 7(16):576-588.

[32] Yang Li, Tao Zhang, Yanhui Zhang, et al. Targeting the FOXM1-regulated long noncoding RNA TUG1 in osteosarcoma. Cancer Sci. 2018, 109(10):3093-3104.

\section{Data Availability}

The datasets used and analyzed in the current study are available from the corresponding author on reasonable request.

\section{Competing Interests}

The authors declare that there are no competing interests associated with the manuscript.

\section{Funding}

Supported by Natural Science Foundation of Liaoning Province of China. 


\section{Author Contributions}

The work presented here was carried out in collaboration between all authors. YW and GZ contributed to the conception of the study; YZ and GY contributed significantly to analysis and manuscript preparation. GY and JG performed the data analyses and wrote the manuscript; YL and LX helped perform the analysis with constructive discussions.

\section{Statement}

Patients have given their written informed consent in our study. And ethics committee of Liaoning cancer hospital approved the study protocol. The study protocol conforms to the ethical guidelines of the 1975 Declaration of Helsinki. I declare that all methods are reported in accordance with ARRIVE guidelines and all methods were carried out in accordance with relevant guidelines and regulations.

\section{Consent to Publication}

All the authors agreed to publish the manuscript.

\section{Acknowledgment}

We appreciate all the participants who provide supports for our research.

\section{Figure legend}

Figure 1 PTTG3P is upregulated in CRC tissues, and cell lines.

$(\mathrm{A}, \mathrm{B})$ Exploring PTTG3P expression in CRC cell lines by assembling the Cancer Cell Line Encyclopedia (CCLE) (www.broadinstitute.org/ccle).

(C) The expression profiles of PTTG3P in HT-29, HCT-8, SW480, HCT116 and FHC was detected with qRT-PCR.

(D,E) qRT-PCR analysis was used to evaluate PTTG3P expression in 120 paired tumor and paired adjacent non-tumor tissues.

$(\mathrm{F}, \mathrm{G})$ High PTTG3P expression was observed in TCGA database of CRC $(\mathrm{n}=521)$.

(H) High PTTG3P expression was observed in TCGA database of malignant tumors.

(I,J) High PTTG3P expression was observed in STAD and ESCA $(n=20)$.

$* \mathrm{P}<0.05,{ }^{* *} \mathrm{P}<0.01, * * * \mathrm{P}<0.001$

Figure 2 Overexpressed PTTG3P correlates with poor prognosis in CRC.

(A) The expression of PTTG3P was checked in different clinical stages of CRC tissues

(B) PTTG3P expression and survival predicted poor prognosis of OS in a cohort of 120 paired cases.

(C) ROC curve of PTTG3P.

(D) Short hairpin RNA (shRNA) targeting PTTG3P and PTTG3P overexpressed plasmids were transfected into HCT116 and HT-29 cells.

(E) GSEA plot showing that PTTG3P expression positively correlated with glycolysis-activated gene signatures (REACTOME GLYCOLYSIS).

(F) Analysis of glycolic gene expression in PTTG3P knockdown or re-expressed PTTG3P.

(G) qPCR analysis showed that PTTG3P was upregulated under low glucose culture conditions compared to normal glucose in a dose-dependent manner and time-dependent manner $(2.5 \mathrm{mM})$. (H) QPCR analysis showed that PTTG3P was increased after 2-DG treatment in dose-dependent manner and in time-dependent manner. 
$* \mathrm{P}<0.05, * * \mathrm{P}<0.01, * * * \mathrm{P}<0.001$

Figure 3 PTTG3P promoted the glycolysis and proliferation of CRC.

(A) Glucose uptake analysis, (B) Lactate production analysis, and (C) ATP analysis explored the glucose uptake, lactate production, and ATP accumulation.

(D) Extracellular acidification rate (ECAR) analysis tested the glycolytic capacity in CRC cells transfectedwith sh-PTTG3P or overexpressed PTTG3P.

(E) CCK8 assay detected the proliferation of HCT116 and HT-29 cells transfected with sh-PTTG3P or overexpressed PTTG3P.

(F) Flow cytometry assays revealed that PTTG3P affected cell apoptosis

(G) Tumor volume and $(\mathrm{H})$ weight were measured in vivo when injected with sh-PTTG3P transfected HCT116 cells.

(I) CCK8 assay detected the proliferation of HT-29 cells transfected with overexpressed PTTG3P and treated with 2.5mM 2-DG or $100 \mu \mathrm{M} 3-\mathrm{BP}$.

(J) Xenograft tumors volume, (K) Xenograft tumors weight were established, with injected with PTTG3P or PTTG3P plus sh-LDHA or PTTG3P treated with 2.5mM 2-DG HT-29 cells . Empty vector as indicated.

(L) Tumor volume and (M) weight were measured in vivo when injected with sh-PTTG3P and oxaliplatin treatment transfected HCT116 cells.

Data are presented as the mean \pm SD from three independent experiments.

$* \mathrm{P}<0.05, * * \mathrm{P}<0.01, * * * \mathrm{P}<0.001$

\section{Figure 4 PTTG3P regulates Hippo signaling pathway in CRC}

(A) GSEA plot showing that PTTG3P expression positively correlated with YAP-activated gene signatures

(B) PTTG3P knockdown impaired the mRNA level of YAP1, FOXM1 and CTGF.

(C) The relationship between PTTG3P and YAP1 was analysed by Spearman's correlation analysis.

(D) CCK8 assay detected the proliferation of HT-29 cells transfected with PTTG3P or PTTG3P plus sh-YAP1.

(E) Flow cytometry assays revealed that PTTG3P plus sh-YAP1 could partly rescue cell apoptosis.

(F) Xenograft tumors volume, $(\mathrm{G})$ Xenograft tumors weight were established, with injected with PTTG3P or PTTG3P plus sh-YAP1. Empty vector as indicated.

(H) CCK8 assay detected the proliferation of HT-29 cells transfected with PTTG3P or PTTG3P plus XMU-MP-1

(I) Flow cytometry assays revealed that PTTG3P plus XMU-MP-1 could partly rescue cell apoptosis.

(J)Xenograft tumors volume, $(\mathrm{K})$ Xenograft tumors weight were established, with injected with PTTG3P or PTTG3P plus XMU-MP-1. Empty vector as indicated.

Data are presented as the mean \pm SD from three independent experiments.

$* \mathrm{P}<0.05, * * \mathrm{P}<0.01, * * * \mathrm{P}<0.001$

Figure 5 Methylation and acetylation have no effect on the expression of PTTG3P.

(A,B)Prediction of $\mathrm{CpG}$ islands in PTTG3P promoter region by analyzing the sequences of PTTG3P promoter through the MethPrimer online software 
(http://www.urogene.org/cgibin/methprimer/methprimer.cgi) and DBCAT

(http://dbcat.cgm.ntu.edu.tw/)

(C)The role of silencing DNMT1, DNMT3A, DNMT3B on the expression of PTTG3P in HT-29 and

HCT116 cells.

(D) qRT-PCR analysis of PTTG3P in HT-29 and HCT116 cells treated with DMSO or 5-AZA (5 $\mu \mathrm{M}$ or $10 \mu \mathrm{M})$ for $72 \mathrm{hr}(\mathrm{n}=3)$.

(E) The broad-spectrum HDAC inhibitors (SAHA and NaB) on the expression of PTTG3P in HT-29 cells.

(F) Overexpressed HDAC6 and HDAC8 on the expression of PTTG3P in HT-29 cells.

(G) The role of silencing EZH2 and LSD1 on the expression of PTTG3P in HT-29 and HCT116 cells.

$* \mathrm{P}<0.05, * * \mathrm{P}<0.01, * * * \mathrm{P}<0.001$

\section{Figure 6 FoxM1 could regulate PTTG3P exopression and abrogate PTTG3P function}

(A) The FoxM1 interference efficiency using 2 different siRNAs and negative control detected by qRT-PCR.

(B) The role of silencing FoxM1 and rescue assay on the expression of PTTG3P in HT-29 and HCT116 cells.

(C) Truncations of PTTG3P promoter were cloned into pGL3-basic vectors, and then luciferase activity was assessed after transfecting these constructed vectors into HEK-293 T cells.

(D) Co-transfection of luciferase reporter containing PTTG3P promoter region $(-1200 \sim 0)$ and $(-900 \sim$ 0), and si-FoxM1 into HEK-293 T cells to test the role of FoxM1 on PTTG3P promoter activity.

(E) ChIP assay was performed to show FoxM1 could directly bind to the PTTG3P promoter region $(-1200 \sim 0)$.

(F, G, H) PTTG3P truncation (-900 0 nt) could barely promote glucose uptake, cell proliferation and inhibited apoptosis.

(I, J) PTTG3P with promoter region $(-900 \sim 0 \mathrm{nt})$ could partly rescue the effect of sh-PTG3P on the downstream genes, with no statistical sinificance.

(K-O) si-FoxM1 rescued PTTG3P bioeffect of cell proliferation and apoptosis.

(P) The graph of mechanism of the study.

$* \mathrm{P}<0.05, * * \mathrm{P}<0.01, * * * \mathrm{P}<0.001$

\section{Figure S1}

(A) According to an online database (GSE84983), the volcano graph showed differential genes.

(B) qRT-PCR of the expressions of the top 5 up-regulated lncRNAs in CRC.

(C) PTTG3P localization was predicted using IncLocator (IncRNA subcellular localization predictor).

(D) PTTG3P localization was validated by subcellular fractionation.

(E-G)The difference in the expression of PTTG3P between CRC samples with somatic mutations in

EGFR, VEGFR or RAS and those without such mutations.

$(\mathrm{H}, \mathrm{I})$ High YAP1 expression was observed in CRC (TCGA-COAD, $\mathrm{n}=521$ ).

(J) ROC curve analysis of the diagnostic value of YAP1.

$* \mathrm{P}<0.05,{ }^{*} * \mathrm{P}<0.01, * * * \mathrm{P}<0.001$

\section{Figure S2}


Developed radiomics nomogram, the radiomics nomogram was developed with tumor differentiation, tumor size, PTTG3p expression and YAP1 expression incorporated.

\section{Figure S3}

(A) ROC curve of FoxM1, PTTG3P and YAP1.

(B) qRT-PCR analysis was used to evaluate FoxM1 expression in 120 paired tumor and paired adjacent non-tumor tissues.

(C,D) High FoxM1 expression was observed in CRC (TCGA-COAD, $\mathrm{n}=521$ ).

(E) High FoxM1 expression was observed in TCGA database of malignant tumors.

$* \mathrm{P}<0.05,{ }^{* *} \mathrm{P}<0.01, * * * \mathrm{P}<0.001$

\section{Figure S4}

(A) The inhibitors of FoxM1 (FDI-6 treatment for 24h) on the expression of PTTG3P in HT-29 and HCT116 cells.

(B) ChIP assay was performed to show FDI-6 (treatment for 12h) could impede FoxM1 binding to the PTTG3P promoter region.

(C) ChIP-PCR showed FDI-6 (treatment for 12h) decreased FoxM1 occupancy at CCNB1 and $\mathrm{CDC} 25 \mathrm{~B}$ promoter region.

(D) The correlation of PTTG3P and immune cell correlation in TCGA-COAD database.

(E) High and low PTTG3P expression with CD8+ T, NK and TFH cells infiltration in TCGA-COAD database.

(F) Scatter plot of PTTG3P expression and CD8 $+\mathrm{T}$ and NK cells infiltration in TCGA-COAD database.

$(\mathrm{G}, \mathrm{H})$ High and low PTTG3P expression with Treg or macrophages infiltration in TCGA-COAD database.

(I) Scatter plot of PTTG3P expression and Treg cells infiltration in TCGA-COAD database.

(J) ELISA detection of the expression of TNF- $\alpha$, IL-1 $\beta$ and IL-6 in cell culture supernatants after depletion of PTTG3P in HCT116 cell.

$* \mathrm{P}<0.05, * * \mathrm{P}<0.01, * * * \mathrm{P}<0.001$ 
A

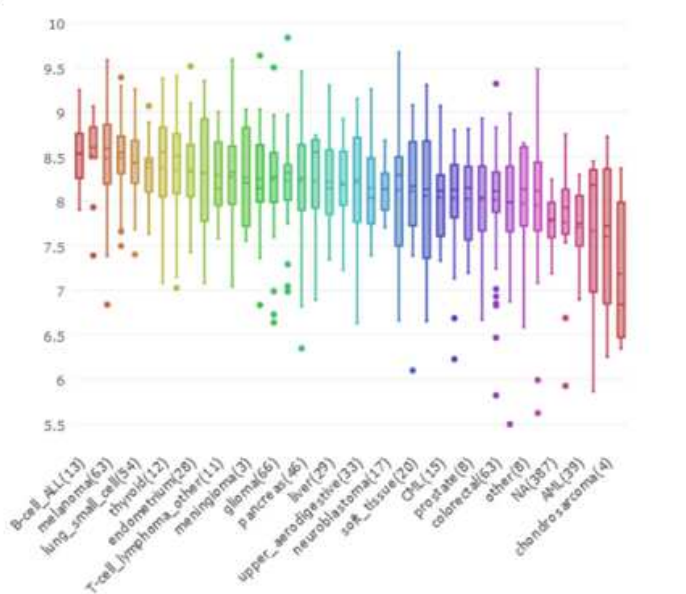

C

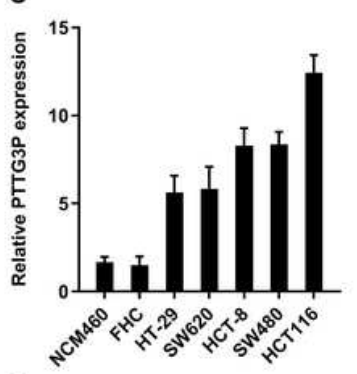

H

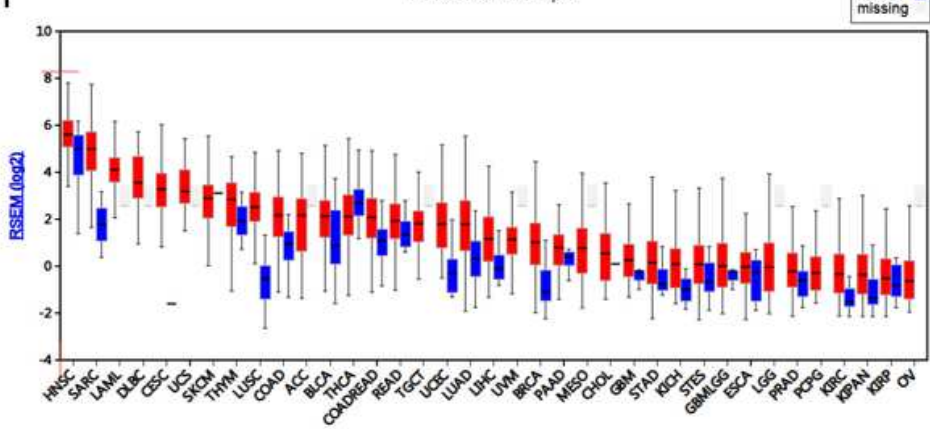

B

E
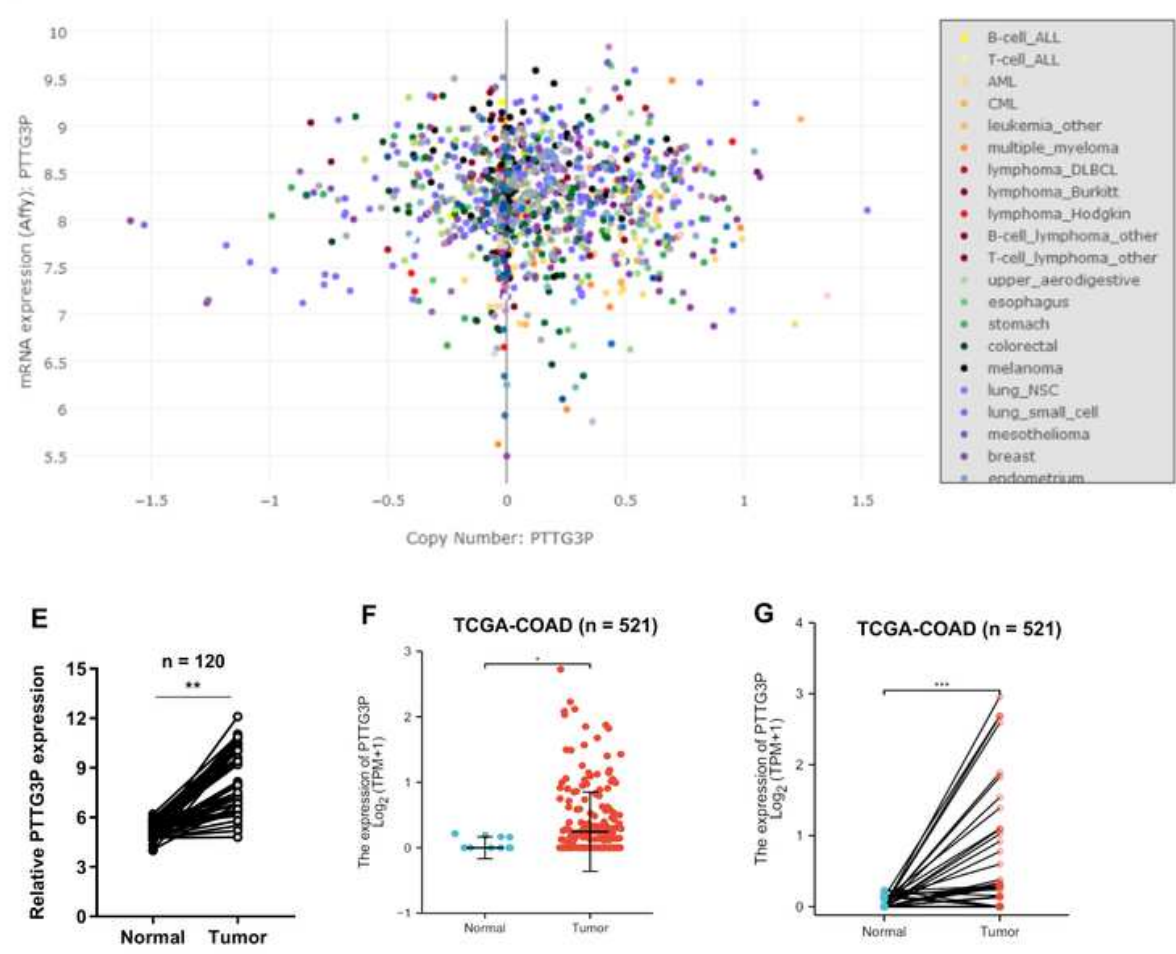

F

Copy Number: PTTG3P
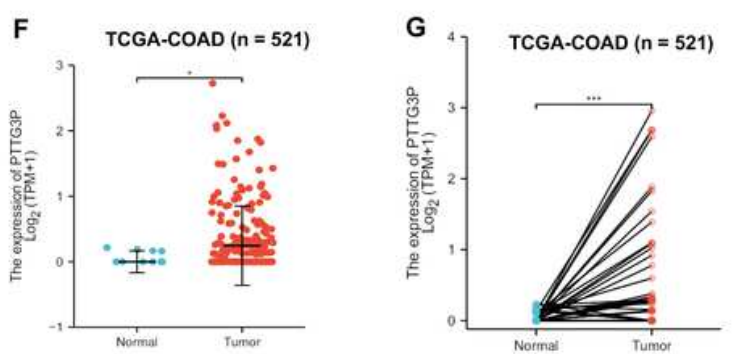

I

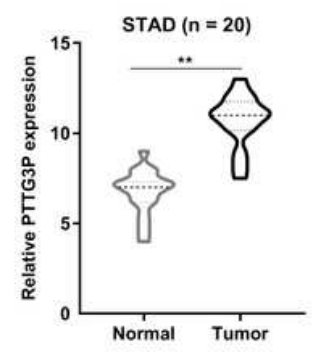

J

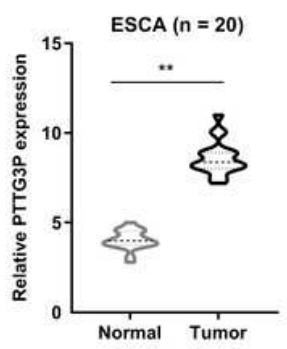

Figure 1

PTTG3P is upregulated in CRC tissues, and cell lines. (A,B) Exploring PTTG3P expression in CRC cell lines by assembling the Cancer Cell Line Encyclopedia (CCLE) (www.broadinstitute.org/ccle). (C) The expression profiles of PTTG3P in HT-29, HCT-8, SW480, HCT116 and FHC was detected with qRT-PCR. $(D, E)$ qRT-PCR analysis was used to evaluate PTTG3P expression in 120 paired tumor and paired adjacent non-tumor tissues. $(F, G)$ High PTTG3P expression was observed in TCGA database of CRC ( $\mathrm{n}=$ 521). (H) High PTTG3P expression was observed in TCGA database of malignant tumors. $(\mathrm{I}, \mathrm{J})$ High PTTG3P expression was observed in STAD and ESCA $(n=20)$. ${ }^{*}<0.05, * \star P<0.01, * \star * P<0.001$ 
A

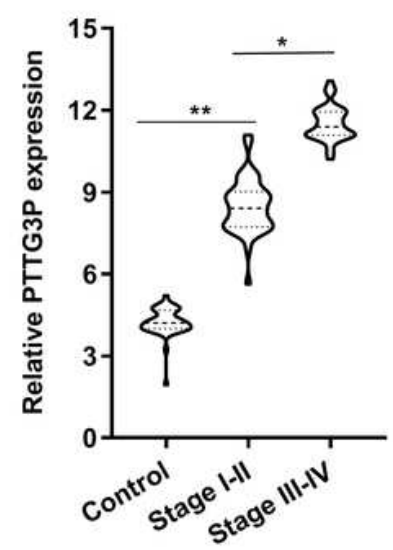

D

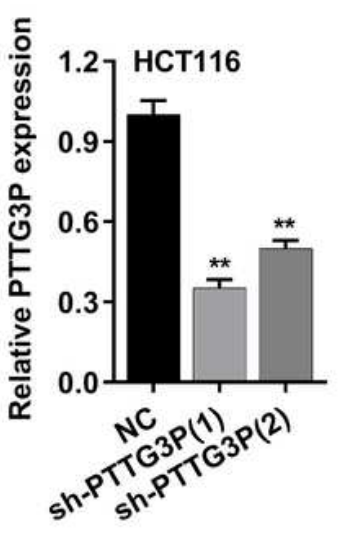

G

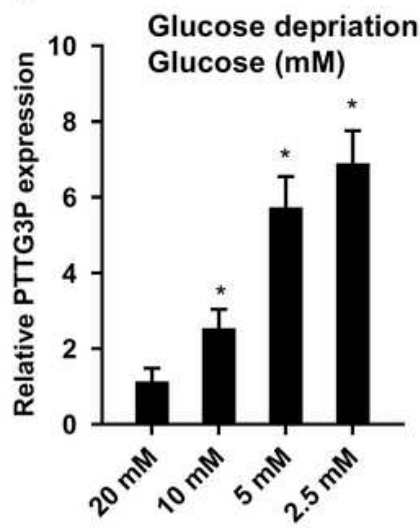

B

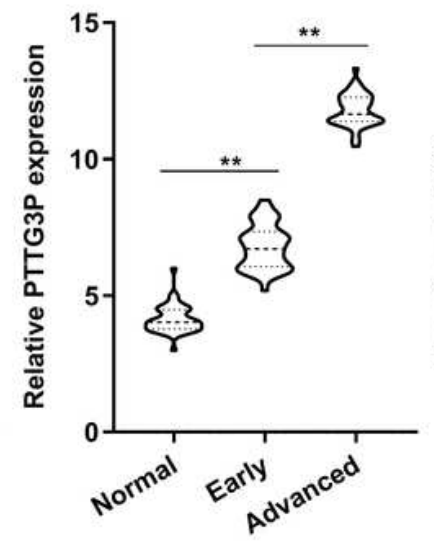

E

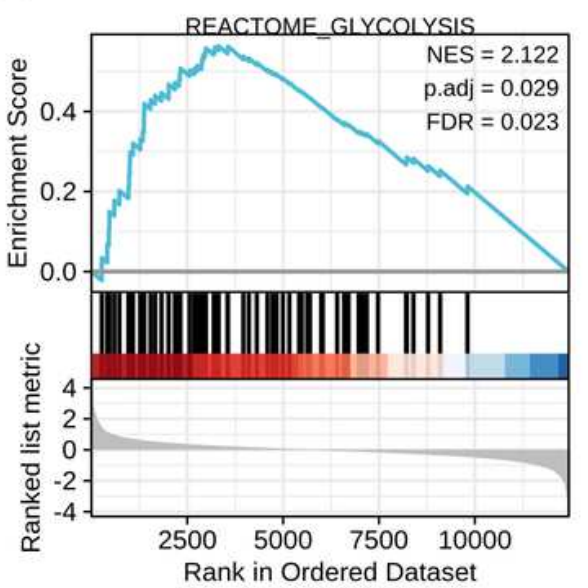

C

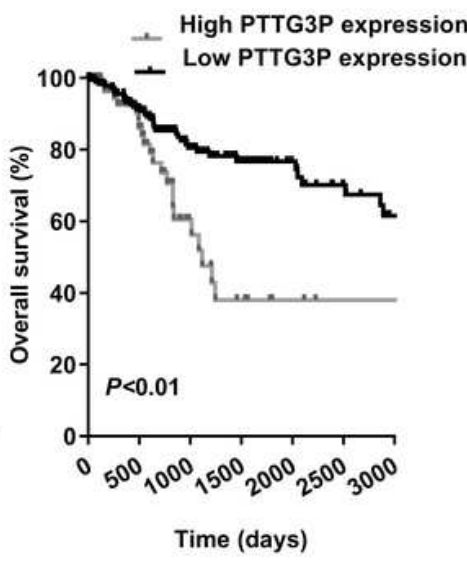

F

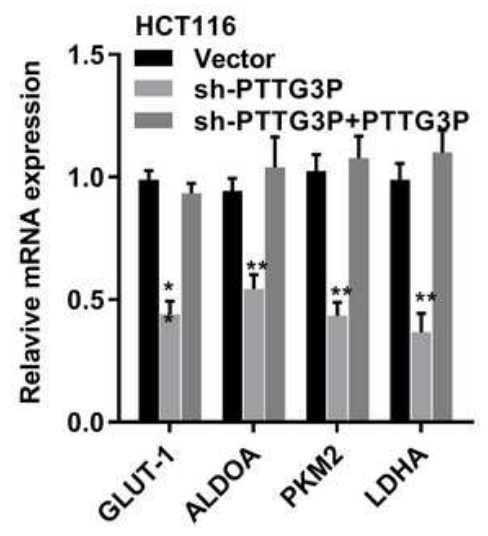

Figure 2

Overexpressed PTTG3P correlates with poor prognosis in CRC. (A) The expression of PTTG3P was checked in different clinical stages of CRC tissues (B) PTTG3P expression and survival predicted poor prognosis of OS in a cohort of 120 paired cases. (C) ROC curve of PTTG3P. (D) Short hairpin RNA (shRNA) targeting PTTG3P and PTTG3P overexpressed plasmids were transfected into HCT116 and HT29 cells. (E) GSEA plot showing that PTTG3P expression positively correlated with glycolysis-activated gene signatures (REACTOME GLYCOLYSIS). (F) Analysis of glycolic gene expression in PTTG3P 
knockdown or re-expressed PTTG3P. (G) qPCR analysis showed that PTTG3P was upregulated under low glucose culture conditions compared to normal glucose in a dose-dependent manner and time-dependent manner (2.5mM). (H) QPCR analysis showed that PTTG3P was increased after 2-DG treatment in dosedependent manner and in time-dependent manner. ${ }^{*} P<0.05$, ${ }^{*} P<0.01, * * * P<0.001$

A

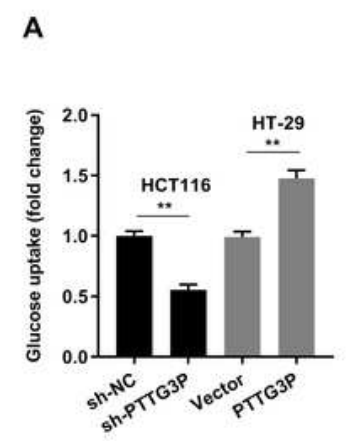

B

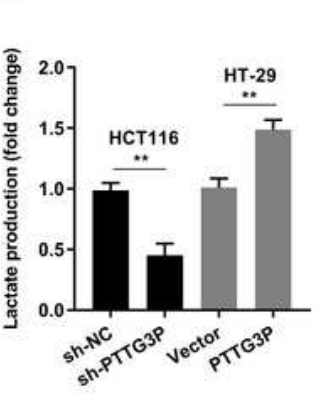

E

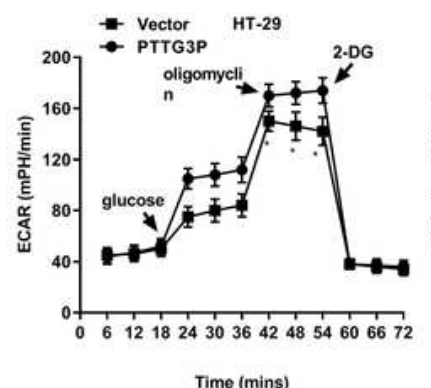

Time (mins)

G

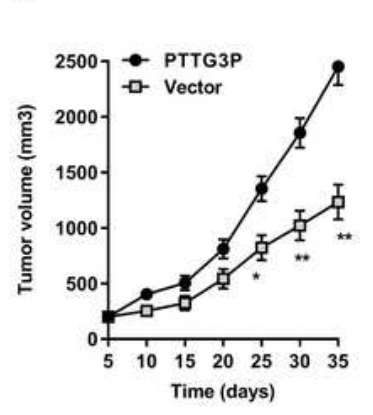

H

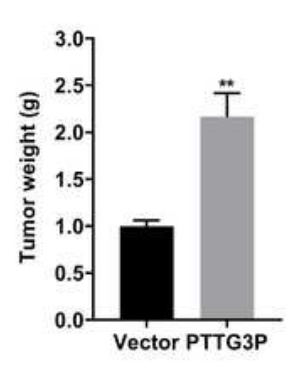

C
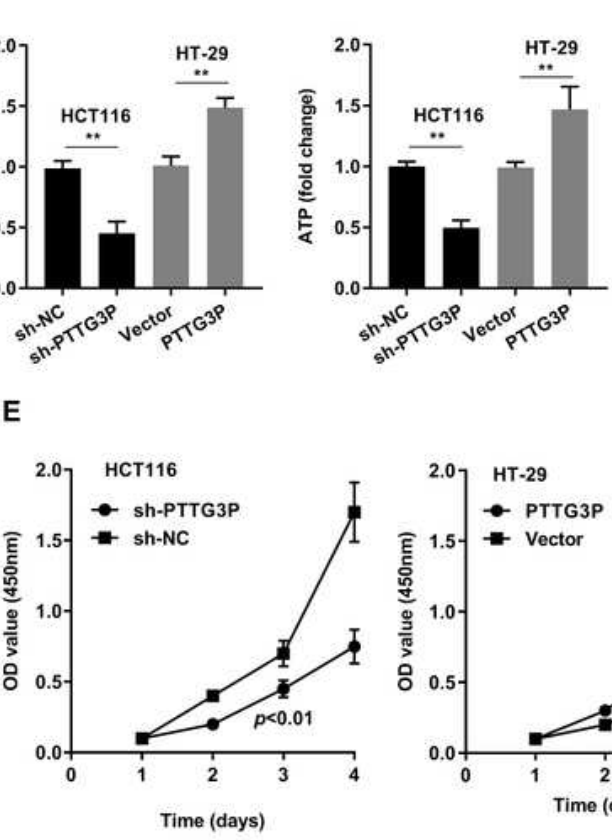

I

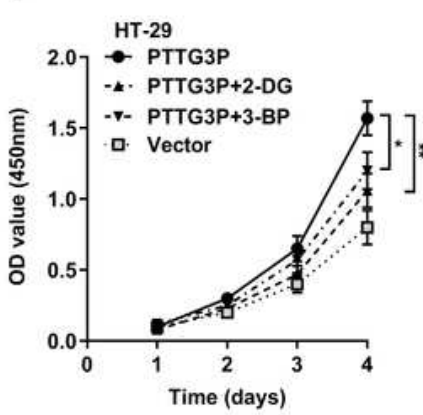

D

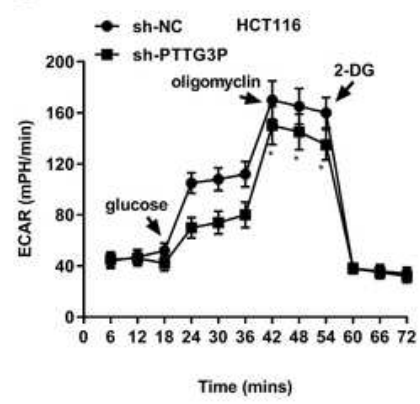

F
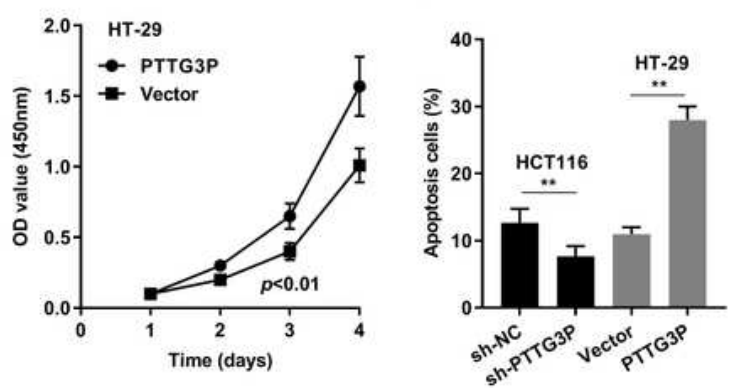

J
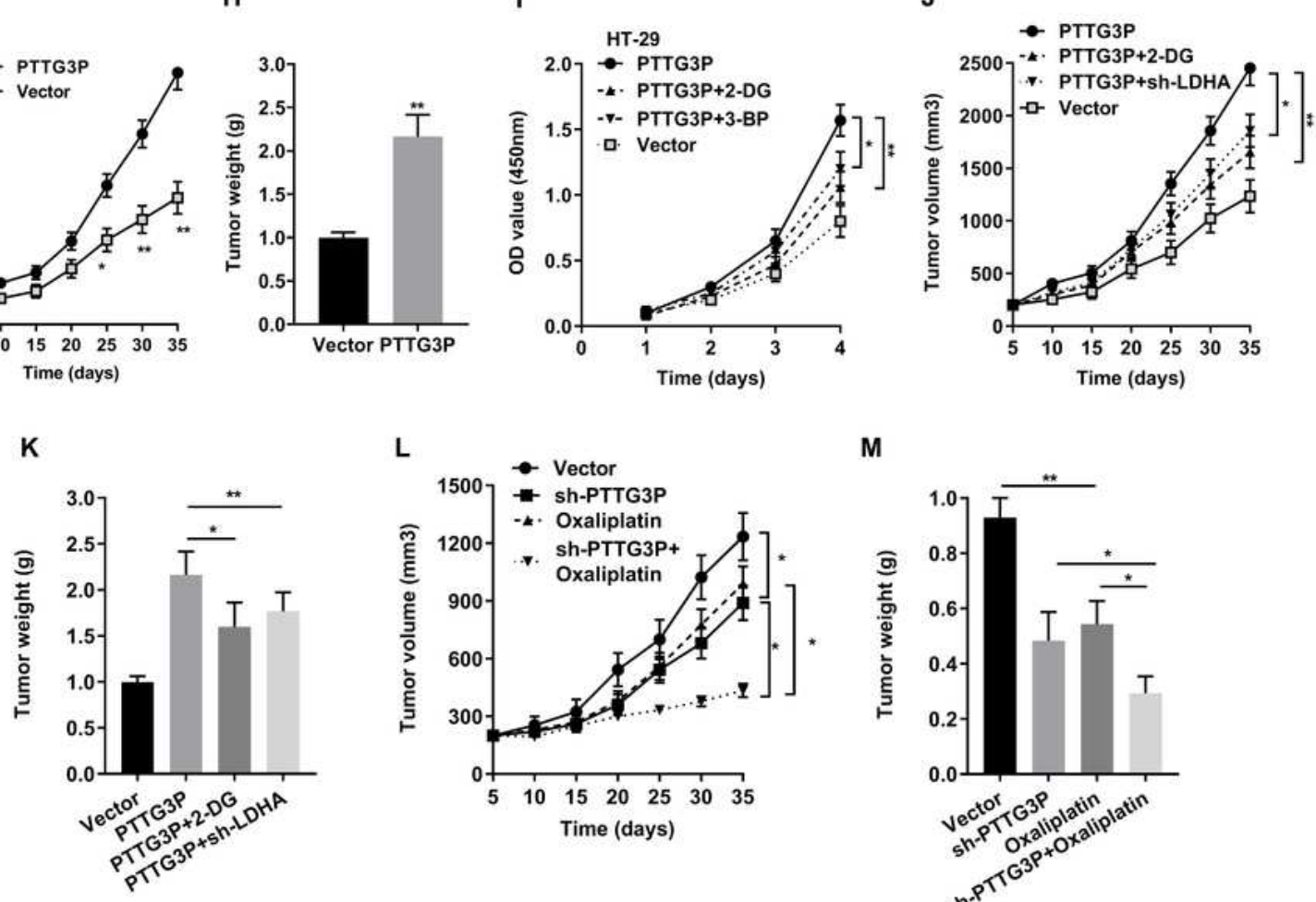

L

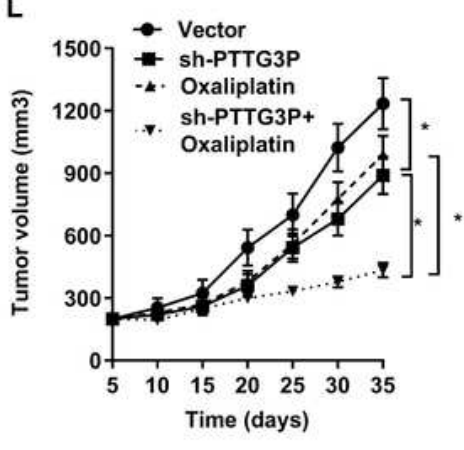

M

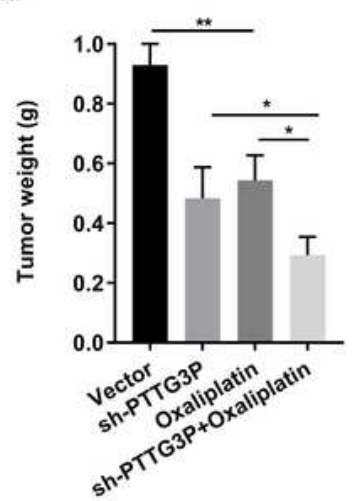

Figure 3 
PTTG3P promoted the glycolysis and proliferation of CRC. (A) Glucose uptake analysis, (B) Lactate production analysis, and (C) ATP analysis explored the glucose uptake, lactate production, and ATP accumulation. (D) Extracellular acidification rate (ECAR) analysis tested the glycolytic capacity in CRC cells transfectedwith sh-PTTG3P or overexpressed PTTG3P. (E) CCK8 assay detected the proliferation of HCT116 and HT-29 cells transfected with sh-PTTG3P or overexpressed PTTG3P. (F) Flow cytometry assays revealed that PTTG3P affected cell apoptosis $(\mathrm{G})$ Tumor volume and $(\mathrm{H})$ weight were measured in vivo when injected with sh-PTTG3P transfected HCT116 cells. (I) CCK8 assay detected the proliferation of HT-29 cells transfected with overexpressed PTTG3P and treated with 2.5mM 2-DG or $100 \mu \mathrm{M}$ 3-BP. (J) Xenograft tumors volume, $(\mathrm{K})$ Xenograft tumors weight were established, with injected with PTTG3P or PTTG3P plus sh-LDHA or PTTG3P treated with 2.5mM 2-DG HT-29 cells . Empty vector as indicated. (L) Tumor volume and (M) weight were measured in vivo when injected with sh-PTTG3P and oxaliplatin treatment transfected HCT116 cells. Data are presented as the mean \pm SD from three independent experiments. ${ }^{*} P<0.05, * \star P<0.01, \star \star * P<0.001$ 
A

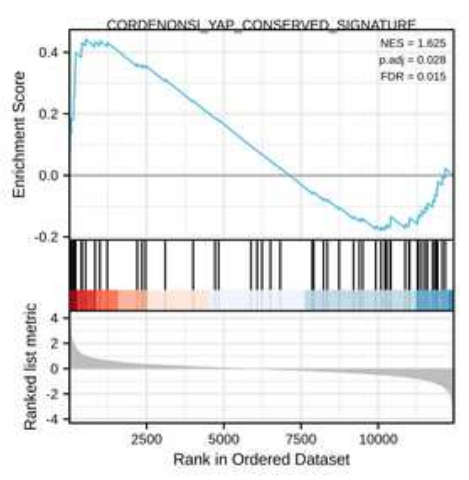

D

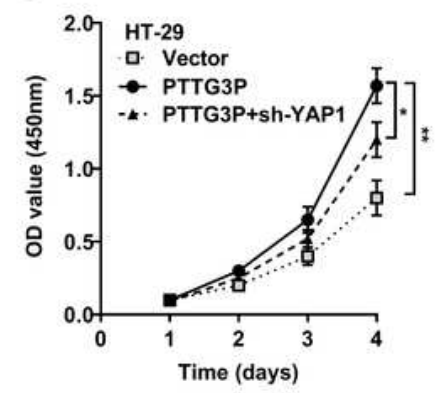

G

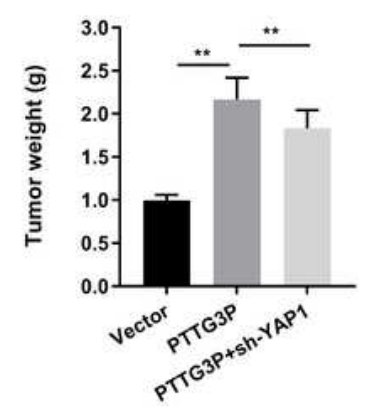

J

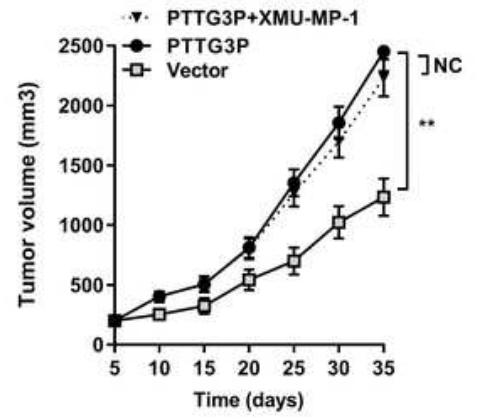

E

H
C
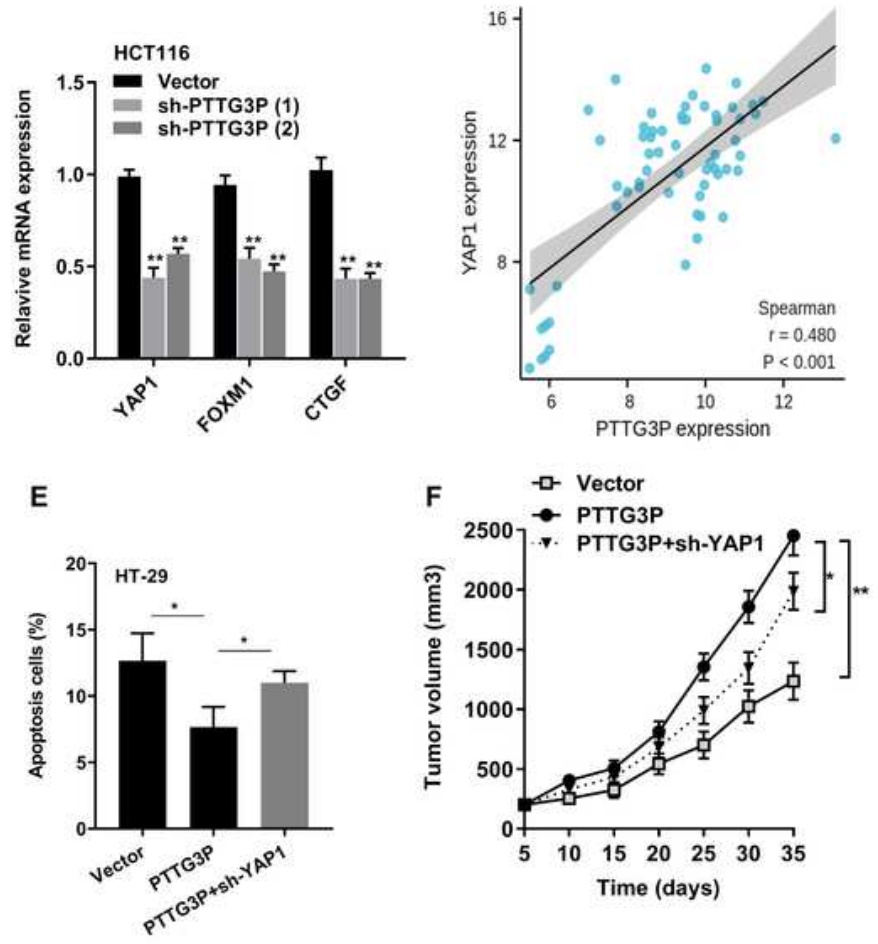

I
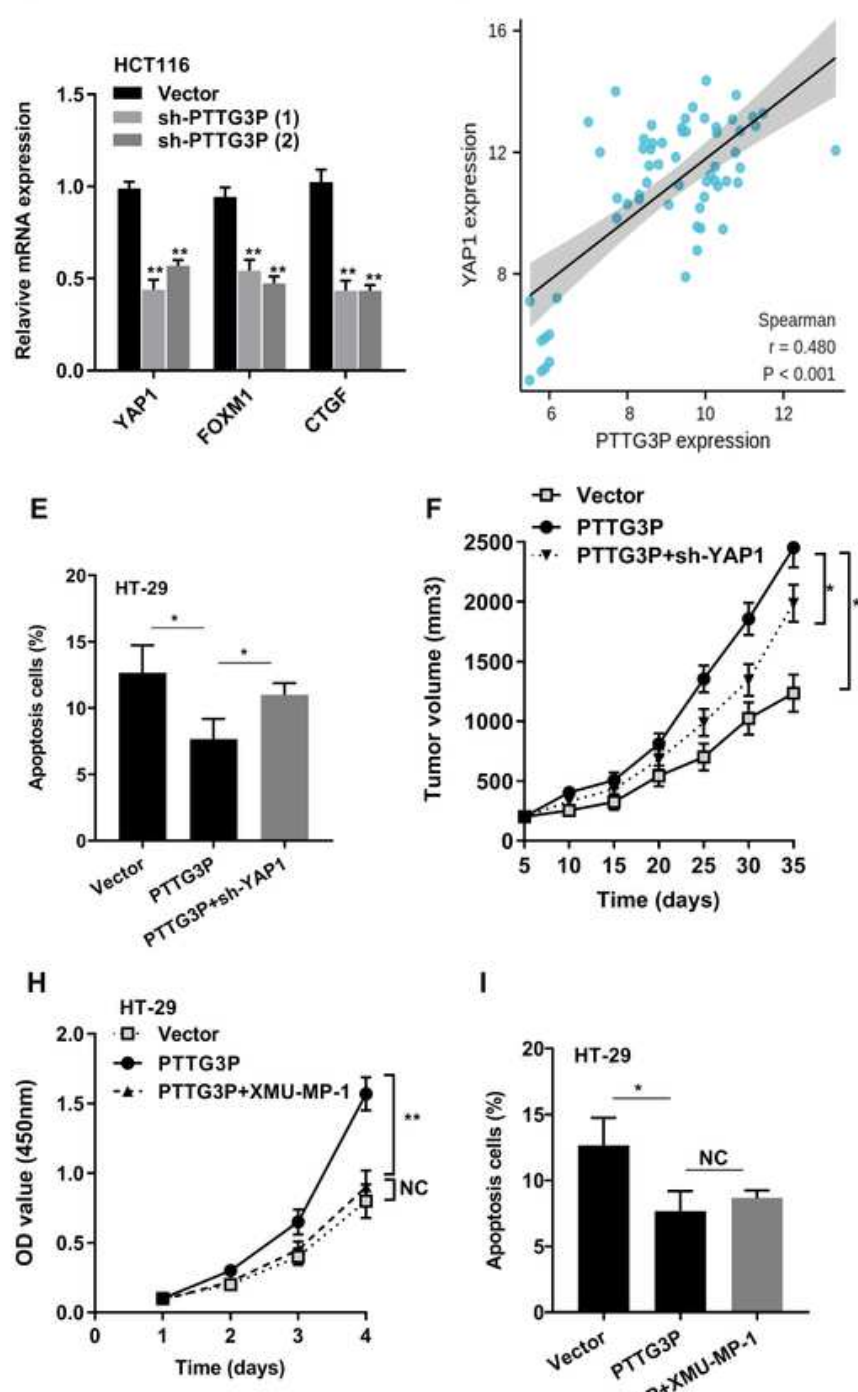

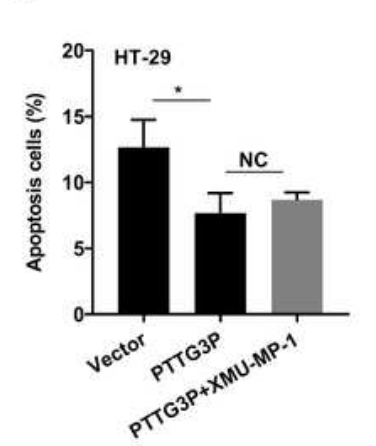

K 
partly rescue cell apoptosis. (F) Xenograft tumors volume, (G) Xenograft tumors weight were established, with injected with PTTG3P or PTTG3P plus sh-YAP1. Empty vector as indicated. (H) CCK8 assay detected the proliferation of HT-29 cells transfected with PTTG3P or PTTG3P plus XMU-MP-1 (I) Flow cytometry assays revealed that PTTG3P plus XMU-MP-1could partly rescue cell apoptosis. (J)Xenograft tumors volume, $(K)$ Xenograft tumors weight were established, with injected with PTTG3P or PTTG3P plus XMUMP-1. Empty vector as indicated. Data are presented as the mean \pm SD from three independent experiments. ${ }^{*} \mathrm{P}<0.05, * * \mathrm{P}<0.01,{ }^{* * *} \mathrm{P}<0.001$

A

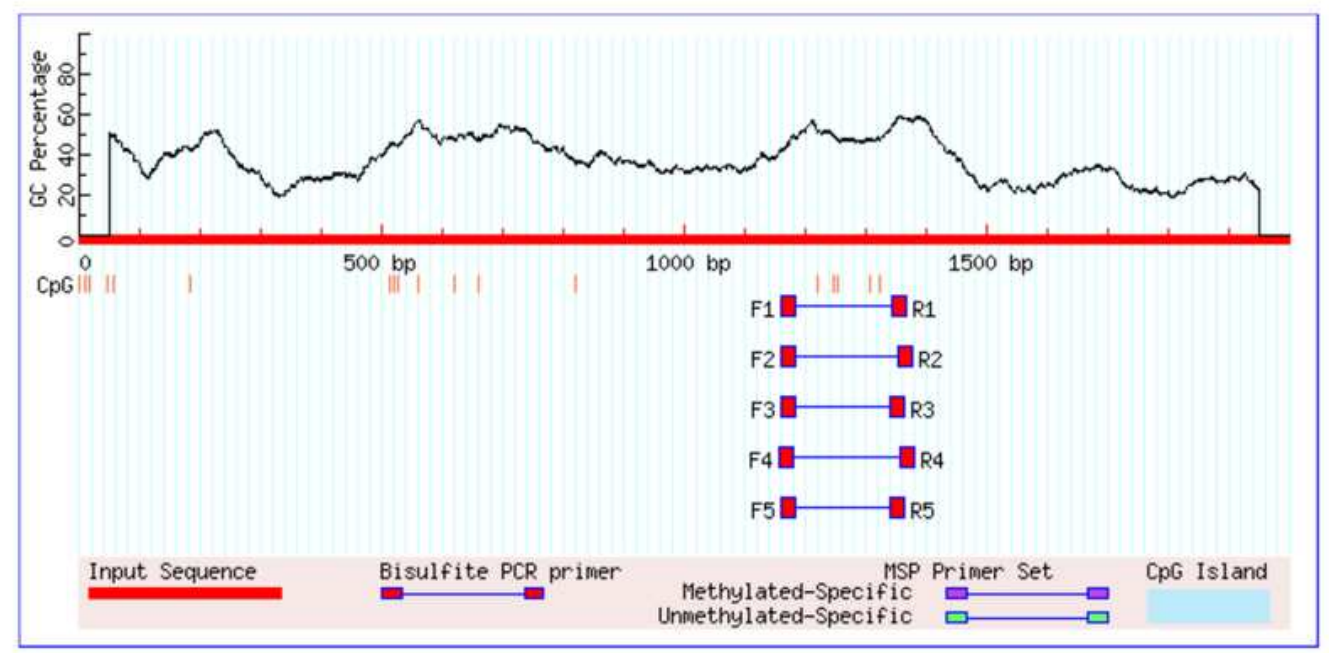

B

- CPG island region CPG site The heaviest CpG density island DNA sequence input

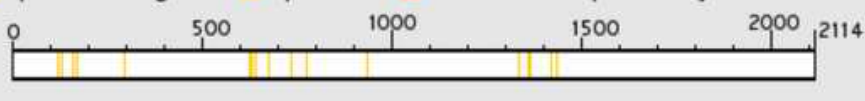

C
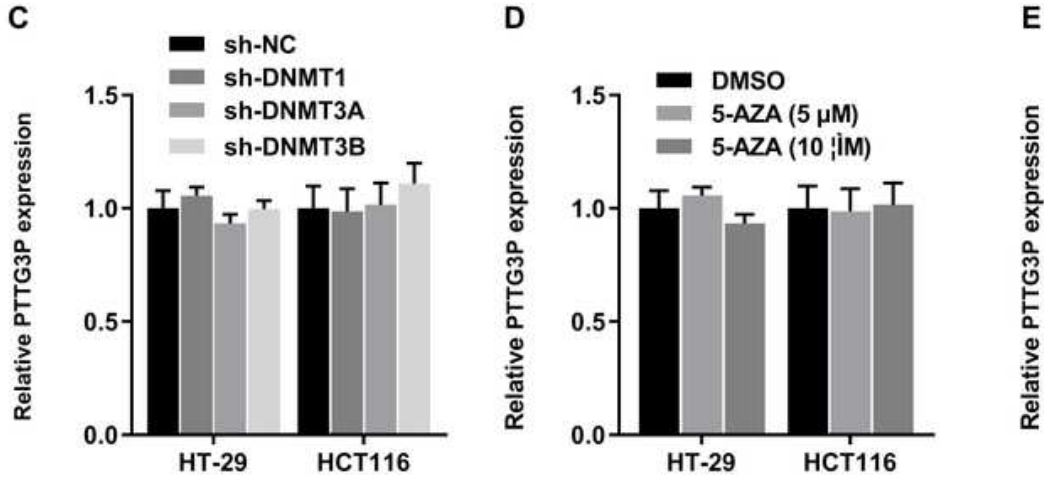

E

F

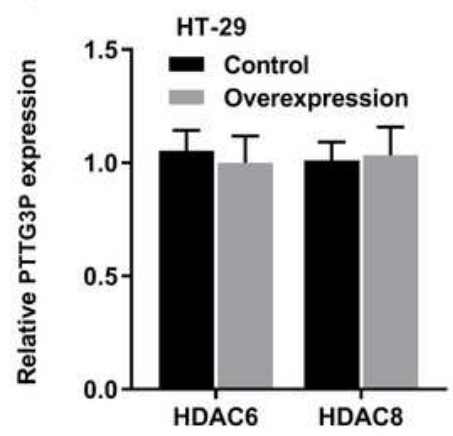

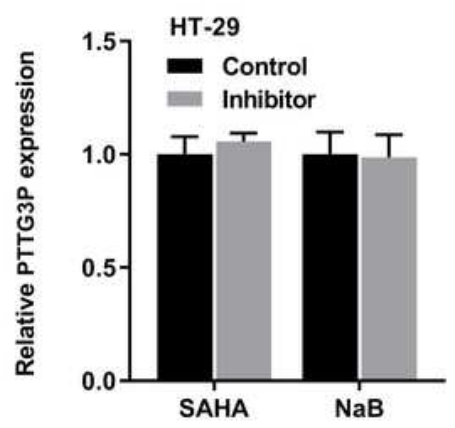

G

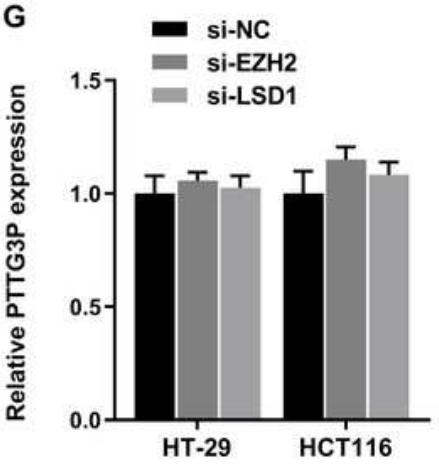




\section{Figure 5}

Methylation and acetylation have no effect on the expression of PTTG3P. (A,B)Prediction of CpG islands in PTTG3P promoter region by analyzing the sequences of PTTG3P promoter through the MethPrimer online software (http://www.urogene.org/cgibin/methprimer/methprimer.cgi) and DBCAT (http://dbcat.cgm.ntu.edu.tw/) (C)The role of silencing DNMT1, DNMT3A, DNMT3B on the expression of PTTG3P in HT-29 and HCT116 cells. (D) qRT-PCR analysis of PTTG3P in HT-29 and HCT116 cells treated with DMSO or 5-AZA ( $5 \mu \mathrm{M}$ or $10 \mu \mathrm{M})$ for $72 \mathrm{hr}(\mathrm{n}=3)$. (E) The broad-spectrum HDAC inhibitors (SAHA and $\mathrm{NaB}$ ) on the expression of PTTG3P in HT-29 cells. (F) Overexpressed HDAC6 and HDAC8 on the expression of PTTG3P in HT-29 cells. (G) The role of silencing EZH2 and LSD1 on the expression of PTTG3P in HT-29 and HCT116 cells. *P $<0.05$, **P $<0.01$, ***P $<0.001$ 
A

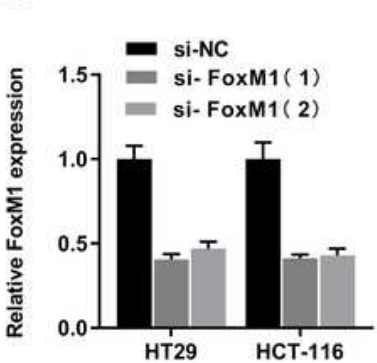

B

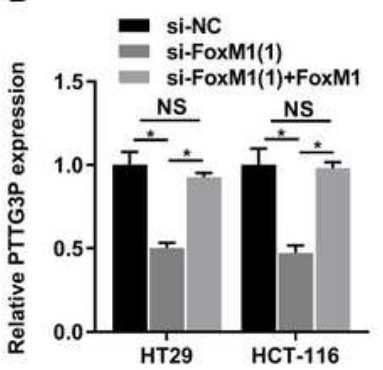

C

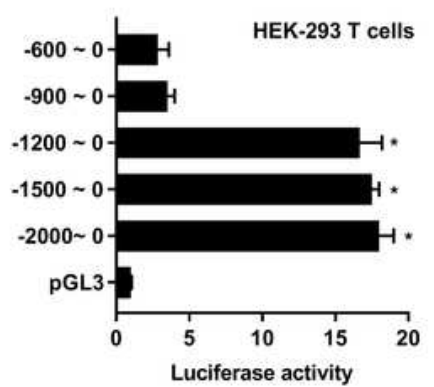

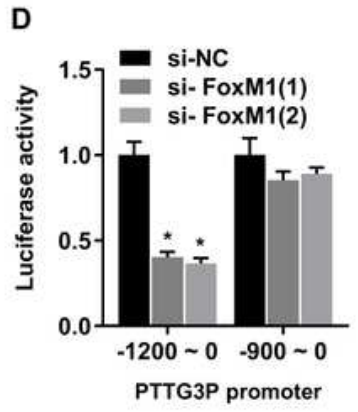

H

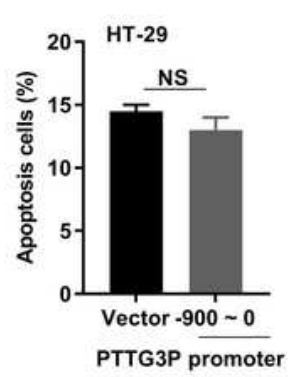

L

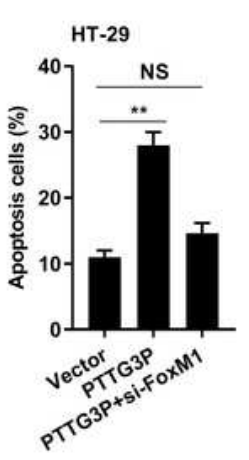

M

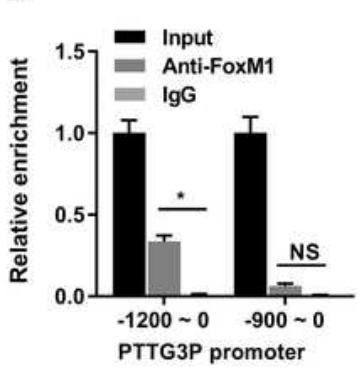

F

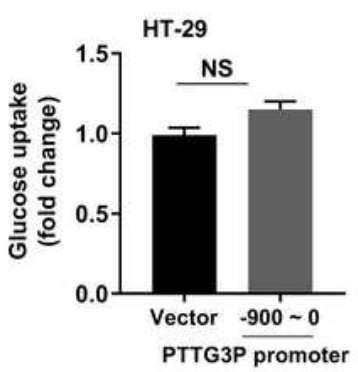

J

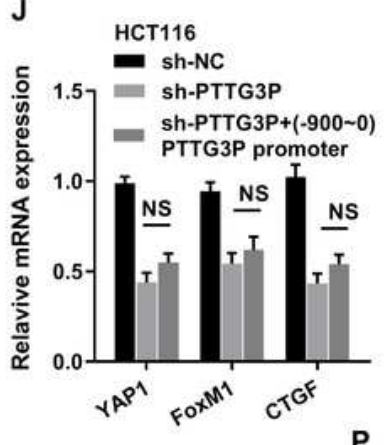

HCT116
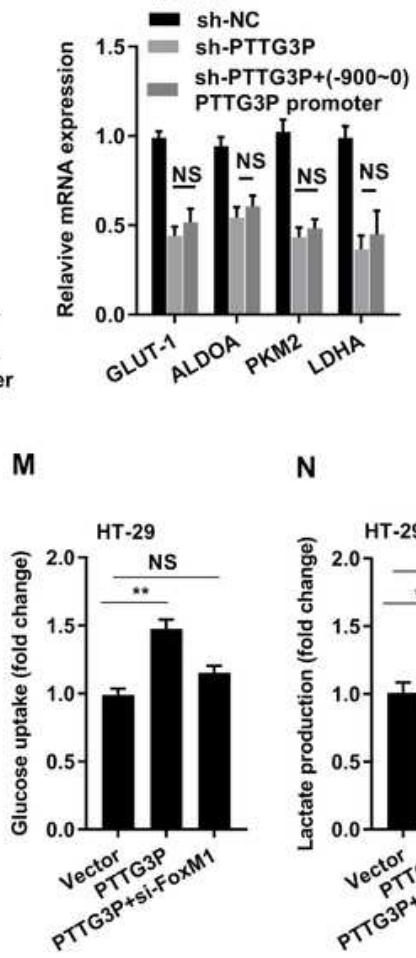

N

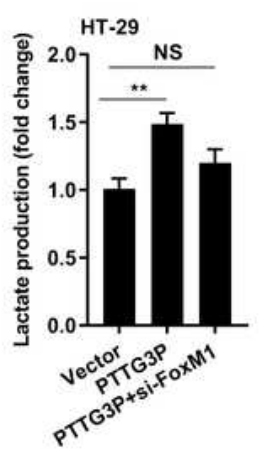

o

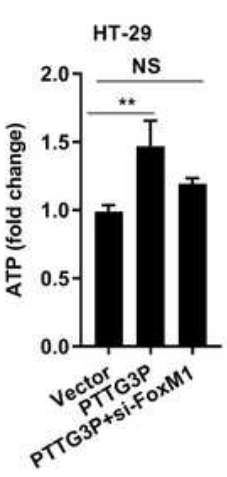

G

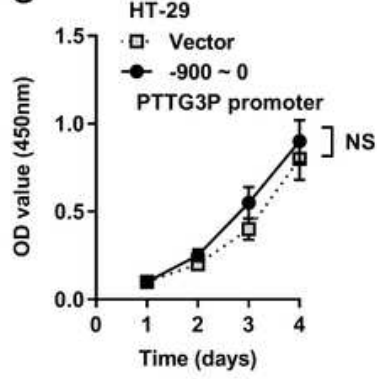

K
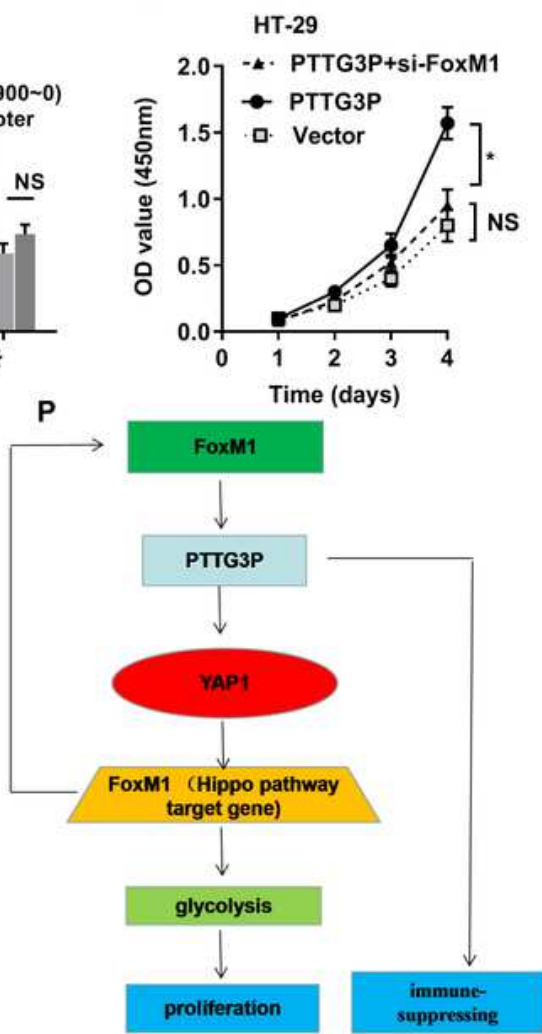

\section{Figure 6}

FoxM1 could regulate PTTG3P exopression and abrogate PTTG3P function (A) The FoxM1 interference efficiency using 2 different siRNAs and negative control detected by qRT-PCR. (B) The role of silencing FoxM1 and rescue assay on the expression of PTTG3P in HT-29 and HCT116 cells. (C) Truncations of PTTG3P promoter were cloned into pGL3-basic vectors, and then luciferase activity was assessed after transfecting these constructed vectors into HEK-293 T cells. (D) Co-transfection of luciferase reporter 
containing PTTG3P promoter region (-1200 0) and (-900 0), and si-FoxM1 into HEK-293 T cells to test the role of FoxM1 on PTTG3P promoter activity. (E) ChIP assay was performed to show FoxM1 could directly bind to the PTTG3P promoter region (-1200 0). (F, G, H) PTTG3P truncation (-900 $0 \mathrm{nt})$ could barely promote glucose uptake, cell proliferation and inhibited apoptosis. (I, J) PTTG3P with promoter region (-900 $0 \mathrm{nt}$ ) could partly rescue the effect of sh-PTG3P on the downstream genes, with no statistical sinificance. (K-O) si-FoxM1 rescued PTTG3P bioeffect of cell proliferation and apoptosis. (P) The graph of mechanism of the study. ${ }^{\star} P<0.05, * \star P<0.01, * \star \star P<0.001$

\section{Supplementary Files}

This is a list of supplementary files associated with this preprint. Click to download.

- figures1.tif

- figureS2Nomogram.tif

- figures3.tif

- figureS4.tif

- supplementarytable.doc 